\title{
Toward trustworthy cloud service selection: A time-aware approach using interval neutrosophic set
}

\author{
Hua Ma ${ }^{a}$, Zhigang Hu ${ }^{\text {a,* }}$, Keqin Li ${ }^{\text {b }}$, Hongyu Zhang ${ }^{c}$ \\ a School of Software, Central South University, Changsha 410075, China \\ ${ }^{\mathrm{b}}$ Department of Computer Science, State University of New York, New Paltz, NY 12561, USA \\ ${ }^{\mathrm{c}}$ School of Business, Central South University, Changsha 410083, China
}

\section{H I G H L I G H T S}

- Propose a time-aware service selection approach for uncertain cloud industry.

- Formulate a multi-criterion decision-making problem using interval neutrosophic set.

- Support tradeoffs between performance-costs and potential risks in time periods.

- Establish the CINS theory to calculate and compare the candidate cloud services.

- Develop a CINS ranking method to create a ranked list of trustworthy cloud services.

\section{A R T I C L E I N F O}

\section{Article history:}

Received 13 October 2015

Received in revised form

12 February 2016

Accepted 9 May 2016

Available online 17 May 2016

\section{Keywords:}

Cloud service selection

Interval neutrosophic set

Performance-costs

Potential risks

Time series analysis

Trustworthy service

\begin{abstract}
A B S T R A C T
Cloud services consumers face a critical challenge in selecting trustworthy services from abundant candidates, and facilitating these choices has become a critical issue in the uncertain cloud industry. This paper employs the time series analysis to address challenges resulting from fluctuating quality of service, flexible service pricing and complicated potential risks in order to propose a time-aware trustworthy service selection approach with tradeoffs between performance-costs and potential risks. The original evaluation data about the services is preprocessed using a cloud model, and interval neutrosophic set (INS) theory is utilized to describe and measure the performance-costs and potential risks of services. In order to calculate and compare the candidate services while supporting tradeoffs between performance-costs and potential risks in different time periods, we established a cloud service interval neutrosophic set (CINS) and designed its operators and calculation rules, with theoretical proofs provided. The problem of time-aware trustworthy service selection is formulated as a multi-criterion decision-making (MCDM) problem of creating a ranked services list using CINS, and it is solved by developing a CINS ranking method. Finally, experiments based on a real-world dataset illustrate the practicality and effectiveness of the proposed approach.
\end{abstract}

(c) 2016 Elsevier Inc. All rights reserved.

\section{Introduction}

\subsection{Motivation}

Recently, cloud computing has been gaining enormous momentum. Cloud service providers around the world have publicized many services [7]. Increasing numbers of cloud service consumers find the convenience and affordability of cloud services alluring;

\footnotetext{
* Corresponding author.

E-mail addresses: hua.ma@csu.edu.cn (H. Ma),zghu@csu.edu.cn (Z.G. Hu), lik@newpaltz.edu (K. Li), hyzhang@csu.edu.cn (H.Y. Zhang).

however, the rapid proliferation of cloud services draws consumers into the dilemma of service selection, especially when multiple services provide similar functionalities. This dilemma has created a critical issue in cloud computing field, that of facilitating cloud service consumers to select trustworthy services with tradeoffs between performance-costs and potential risks [29] among abundant candidates. This task includes the following challenges:

(1) The quality of service (QoS) of cloud services generally fluctuates within a certain range due to the dynamic cloud environment. The quality of experience [27] for consumers is often different from the QoS claimed by service providers. According to the evaluation reports [80] of cloud hosts in China, the performance of services varies widely. In some key 
indicators, such as network throughput and memory speed, the maximum measured value is ten times more than the minimum. The real QoS experienced by consumers can be influenced by factors such as client device type, network location and context $[54,83,77]$. In previous experiments [40] based on real-world WS-DREAM dataset \#2 [88], we analyzed the response time for 5,825 services collected from 339 users, and found that the coefficients of variation for most of services exceed 1.0. As these data illustrate, how to accurately measure the uncertainty of QoS combining the feedback data [40] from consumers, continuous monitoring data [15] or auditing data [36] from service providers, and continuous evaluation data from third parties $[80,12,10]$, has become a key problem in the cloud environment.

(2) Although the load-balancing strategies have been implemented in cloud platforms [34], the load conditions for cloud services may be quite different from one another based on different networks or geographical locations. Within a specified time period, some services are possible under a heavy load, while others might only be possibly under a light load, giving consumers different quality of experience. In order to balance loads, improve energy efficiency and maximize profits, service providers are likely to adopt flexible pricing over time for cloud services [78,48,53]. For example, a service might offer a considerable discount during its light load time period to attract more consumers, or charge additional fees during a time period that is highly volatile in user interaction. Moreover, consumers might in practice attach particular importance to specific time periods according to their application requirements, or purchase a service despite its poor performance during time periods that are less important to them. Therefore, identifying the different performance and cost of service in time periods is of great significance for consumers distinguishing among candidates.

(3) Over the past few years, security problems have increasingly emerged in cloud services, such as Salesforce services, EC2 services, BPOS services, SONY Playstation services and iCloud services. These events have proven that cloud computing is fraught with potential risks that must be carefully evaluated prior to engagement [19]. Recently the risk assessment in cloud services has attracted concern from some organizations, including Cloud Security Alliance (CSA) [13], China Cloud Computing Promotion and Policy Forum (3CPP) [10], and researchers [81,22]. Some primary potential risks to cloud services have been identified and analyzed [17,32]. Especially, recognized as the important risks inherent to the cloud, the availability $[6,56]$ of cloud services and the disruption or failure of cloud computing network [49] are vulnerable to the heavy load and networks' susceptibility in specified time periods. In contrast to performance and costs of cloud services, these potential risks inherent to the cloud are more uncertain. The assessment of potential risks over multiple time periods adds extra complexity to trustworthy service selection problem.

In real-world applications, both the performance-costs and some potential risks of cloud services are dynamic and uncertain during different time periods. Naturally, consumers hope to select the most trustworthy cloud service among abundant candidates by considering the tradeoffs between performance-costs and potential risks over multiple time periods. Consumers may pay less attention to potential risks when performance-cost is more important to them, and may pay less attention to performance-cost during times of sensitivity to potential risks. In order to achieve higher performance-costs in cloud services, consumers can change the time periods of their usage, or they may pay more to enjoy better performance during specific time periods. This paper targets the research tasks of (1) accurately describing and measuring the performance-costs and potential risks of cloud services as a whole, with consideration of uncertainty, and (2) calculating and comparing candidate services with tradeoffs between performance-costs and potential risks, according to the requirements of different application scenarios during different time periods.

\subsection{Our contributions}

In this paper, to measure the uncertainty of cloud environment, we have adopted a new theory known as interval neutrosophic set (INS) [67,84], which is a generalization of classical, three-valued and fuzzy logic. The assessment data of cloud services and the user's application requirements are integrated and transformed into INS. Every service is measured from three aspects, namely performance-costs, potential risks and their uncertainty, which are equivalent to the truth-membership, falsity-membership and indeterminacy-membership, respectively, in INS. The services with high performance-costs, low risks and uncertainty may become the trustworthy candidates. Our strategy for selecting trustworthy services from an abundant field of candidates involves formulating the problem of time-aware service selection with tradeoffs between performance-costs and potential risk as a multicriterion decision-making (MCDM) problem that creates a ranked services list using INS theory.

The main contributions of this paper are as follows:

(1) In order to measure the uncertainty of cloud services and compare candidate services with tradeoffs between performance-costs and potential risks, we propose the cloud service interval neutrosophic set (CINS), based on INS and combining the time period features of cloud services with the tradeoff coefficients from consumers. The aggregation operators and the entropy weight measure method for CINS are designed based on theoretical proofs.

(2) We describe and assess the performance-costs and potential risks of cloud services by utilizing the cloud model and INS theory from the new perspective of time series analysis. Based on this assessment, we formulate the time-aware trustworthy cloud service selection problem with tradeoffs between performance-costs and potential risks over multiple time periods as a MCDM problem employing CINS theory. We then develop a CINS ranking approach to solve the MCDM problem.

(3) We examine the proposed approach through experiments on a real-world dataset and an appropriate baseline for our comparative analysis. Results demonstrate that our approach can work effectively in the risk-sensitive service selection mode and the performance-cost-sensitive service selection mode, and also prevent malignant price competition launched by some low-quality services. This paper can provide decision support approach for time-aware trustworthy service selection problem.

The rest of this paper is organized as follows. Section 2 briefly introduces the related work. Section 3 defines neutrosophic set (NS), INS and CINS. Section 4 defines the problem. Section 5 presents the aggregation operators and the entropy weight measure method for CINS. Section 6 puts forward the time-aware trustworthy service selection approach. Section 7 analyzes the experiments and results. Finally, Section 8 presents conclusions.

\section{Related works}

\subsection{Trustworthy cloud services selection}

Facilitating users' selection of trustworthy candidates from a set of functionally equivalent cloud services represents an exciting 
area of expansion for cloud computing research. Some approaches can provide effective decision supports for this problem as follows:

(1) Approaches based on MCDM methods. MCDM is concerned with structuring and solving decision problems involving multiple criteria. Typically, there is not a unique optimal solution for them and it is necessary to use decisionmaker's preferences to differentiate between solutions. MCDM methods can be used to solve the service selection problem, provided that the trustworthiness attributes and service candidates are finite. Techniques such as the analytic hierarchy process (AHP), analytic network process (ANP), fuzzy analytic hierarchy process (FAHP), ELECTRE and TOPSIS fall into this category. Godse et al. [20] presented an AHP-based SaaS service selection approach to score and rank services objectively; the incorporation of AHP can ameliorate judgmental product prioritization, making the process more rational than strictly subjective ratings. Garg et al. [18] employed an AHP method to measure attributes of QoS and rank cloud services. Similarly, Menzel et al. [43] introduced an ANP method for selecting IaaS services. Ma et al. [40] proposed a trustworthy cloud service selection approach that employs the FAHP method to calculate the weights of user features. Silas et al. [61] developed a cloud service selection middleware based on the ELECTRE method. Sun et al. [63] presented a multi-criteria decisionmaking technique based on fuzzy TOPSIS method to rank cloud services.

(2) Approaches based on prediction. These approaches focus on how to predict the QoS of service accurately and select trustworthy service for users. Techniques such as the probability theory, fuzzy theory, evidence theory, social network analysis (SNA), etc., fall into this category. Mehdi et al. [42] presented a QoS-aware approach based on probabilistic models to assist the service selection, which allows consumers to maintain a trust model of each service provider they have interacted with for the prediction of the most trustworthy service. Qu et al. [51] proposed a system that evaluates trustworthiness of cloud services according to users' fuzzy QoS requirements and services' dynamic performances to facilitate service selection. Huo et al. [26] presented a fuzzy trustworthiness evaluation method combining Dempster-Shafer theory to solve the synthesis of evaluation information for cloud services. Mo et al. [44] put forward a cloud-based mobile multimedia recommendation system by collecting the user contexts, user relationships, and user profiles from video-sharing websites for generating recommendation rules. Targeting the objective and subjective characteristics of trustworthiness evaluations, Ding et al. [16] presented a trustworthiness evaluation framework of cloud services to predict QoS and customer satisfaction for selecting trustworthy services.

(3) Approaches based on recommendation system technologies. These approaches exploit user preferences from history data and achieve personalized service recommendation. By integrating recommendation system technologies such as the collaborative filtering algorithm (CFA), service recommendations based on user feedback have become the dominant trend in trustworthy service selection. Ma et al. [38] presented a user preferences-aware recommendation approach for trustworthy cloud services, in which user preferences are identified by usage preference, trust preference and cost preference. Rosaci et al. [54] proposed an agent-based architecture to recommend multimedia services by integrating the content-based recommendation method and CFA. Wang et al. [66] presented a cloud service selection model employing service brokers to perform dynamic service selection based on an adaptive learning mechanism. Ma et al. [39] proposed a trustworthy service recommendation approach based on interval numbers of four parameters by employing the similarity of client-side feature between potential user and consumers. In order to improve the prediction accuracy of CFA, Hu et al. [25] accounted for the factor of time by proposing a time-aware CFA to predict missing QoS values; this approach collects users' historic data about service at different time intervals and uses it to compute the degree of similarity between services and users. Zhong et al. [89] also proposed a time-aware service recommendation approach by extracting the time sequence of topic activities and the service-topic correlation matrix from service usage history, and forecasting topic evolution and service activity in the near future.

(4) Approaches based on reputation mechanism. The trustworthiness of cloud services can affect the reputation of the service provider; in turn, a reputable service provider is more likely to produce highly trustworthy services. Therefore, evaluating and measuring the reputations of cloud service providers can aid in selecting trustworthy cloud services based on history, expertise and third-party data relevant to cloud service providers. Ramaswamy et al. [52] discussed an approach that utilizes the penalties, prize points and monitoring mechanism of mobile agents to ensure trustworthiness among the cloud broker, customer and service provider. Mouratidis et al. [45] presented a framework incorporating a modeling language that supports the elicitation of security and privacy requirements for selecting suitable service providers. Ayday et al. [5] incorporated belief propagation algorithm to evaluate reputation management systems, and employed factor graph to describe the interactive behavior between consumers and service providers. Pawar et al. [46] proposed an uncertainty model that employs subjective logic operators to calculate the reputations of service providers. Shen et al. [59] proposed a collaborative cloud computing platform, which incorporates multi-faceted reputation management, resource selection, and price-assisted reputation control.

\subsection{Neutrosophic set theory}

Since Zadeh proposed fuzzy set (FS) theory in 1965, many novel extensions have been proposed to settle issues surrounding imprecise, incomplete and uncertain information. These include the interval-valued fuzzy set (IVFS) [65], intuitionistic fuzzy sets (IFS) [3], interval-valued intuitionistic fuzzy sets (IVIFS) [4] and hesitant fuzzy sets (HFS) [64]. In classical set theory, an entity may have a certain degree of membership belonging to a set. As an extension, fuzzy set theory permits the gradual assessment of the membership of elements in a set; this is described with the aid of a membership function valued in the real unit interval $[0,1]$. It also has a certain degree of non-membership, which is taken into consideration in IFS. In addition, HFS was introduced in order to cope with the situations that people are hesitant in expressing their preference over objects in a decision making process. Moreover, the applications of the extensions of FS in various fields have attracted considerable researchers' attention [28,60,47,69,68].

Based on the fact that IFSs cannot handle indeterminate information - that is, the zone of ignorance for a proposition's value between truth and falsity [74] - Smarandache [62] proposed neutrosophic logic and the neutrosophic set (NS). As the truthmembership, indeterminacy-membership, and false-membership in NS are independent, it is a set in which each element of the universe possesses degrees of truth, indeterminacy and falsity, whose values lie in the non-standard unit interval $] 0^{-}, 1^{+}[$, which is an extension to IFS's standard interval $[0,1]$. The uncertainty involved here, that is, the indeterminacy factor, is independent of truth and falsity values. NS has been used in a variety of fields, including intrusion detection systems [31], image segmentation [58,23,21, 
85], artificial intelligence [2], growth and decline analysis of new economies [1] and financial dataset detection [33].

For the convenience of application of NS in practical application, Wang et al. [67] proposed an instance of NS called a singlevalued neutrosophic set (SVNS). In turn, Ye [75] put forward a simplified neutrosophic set (SNS), which can be described by three real numbers in the real unit interval $[0,1]$. Sometimes the degrees of truth, falsity and indeterminacy in a certain statement cannot be precisely defined in real situations, but they can be denoted by several possible interval values, requiring the interval neutrosophic set (INS). Wang et al. [67] proposed the concept of INS and provided its set-theoretic operators.

NS has also been applied to MCDM problems. Ye [74] developed a MCDM approach using a SVNS correlation coefficient measurement. Zhang et al. [82] presented a new correlation coefficient measure of INS and a MCDM method is developed, which takes into account the influence of the evaluations' uncertainty and both the objective and subjective weights. In another study, Liu et al. [37] presented several novel SVNS aggregation operators based on Hamacher operations and developed a multi-criteria group decision-making approach. To address the situations that the criteria are not independent and subject to compensation, Zhang et al. [84] presented a outranking approach based on INS and ELECTRE IV for MCDM problems. Şahin et al. [55] proposed a MCDM method based on inclusion measure for INS.

To the best of our knowledge, no similar research has investigated cloud service interval neutrosophic set (CINS) theory and the MCDM method of modeling a trustworthy cloud service selection problem with tradeoffs between performance-costs and potential risks from the perspective of time series analysis.

\section{Preliminary concepts}

\subsection{IN, NS, INS and their operators}

This section introduces some basic concepts and definition utilized in the rest of the paper.

Definition 1 ([57,8,72]). Let $\tilde{a}=\left[a^{L}, a^{U}\right]=\left\{a^{L} \leq x \leq a^{U}\right\}$; then $\tilde{a}$ is termed as an interval number (IN). If $0 \leq a^{L} \leq x \leq a^{U}$, then $\tilde{a}$ is a positive interval number.

Definition 2 ([71]). Let $\tilde{a}=\left[a^{L}, a^{U}\right], \tilde{b}=\left[b^{L}, b^{U}\right], l_{\tilde{a}}=a^{U}-a^{L}$ and $l_{\tilde{b}}=b^{U}-b^{L}$; then the degree of possibility of $\tilde{a} \geq \tilde{b}$ is formulated by Eq. (1):

$P(\widetilde{a} \geq \widetilde{b})=\min \left\{\left|l_{\tilde{a}}+l_{\tilde{b}}\right|, \max \left(a^{U}-b^{L}, 0\right)\right\} /\left(l_{\tilde{a}}+l_{\tilde{b}}\right)$.

Suppose that there are $n$ interval numbers $\tilde{a}_{i}=\left[a_{i}^{L}, a_{i}^{U}\right](i=$ $1,2, \ldots, n)$, and each interval number $\tilde{a}_{i}$ is compared with all interval numbers $\tilde{a}_{j}(j=1,2, \ldots, n)$ using Eq. (1). Then a complementary matrix, $P=\left\{p_{i j}\right\}$, can be constructed. $p_{i j} \geq 0, p_{i j}+$ $p_{j i}=1, p_{i i}=0.5$. On the basis of $P$, the sort value of each interval number $\tilde{a}_{i}$ can be calculated by Eq. (2):

$o_{i}=\left(\sum_{j=1}^{n} P_{i j}+\frac{n}{2}-1\right) /(n(n-1)), \quad i=1,2, \ldots, n$.

Definition 3 ([62]). Let $X$ be a space of points or objects, with a generic element in $X$ denoted by $x$. A NS $A$ in $X$ is described using a truth-membership function $T_{A}(x)$, an indeterminacy-membership function $I_{A}(x)$ and a falsity-membership function $F_{A}(x) . T_{A}(x), I_{A}(x)$ and $F_{A}(x)$ are real standard or nonstandard subsets of $] 0^{-}, 1^{+}[$; that is, $\left.T_{A}(x): X \rightarrow\right] 0^{-}, 1^{+}\left[, I_{A}(x): X \rightarrow\right] 0^{-}, 1^{+}\left[\right.$and $F_{A}(x): X \rightarrow$ ] $0^{-}, 1^{+}\left[\right.$. There is no restriction on the sum of $T_{A}(x), I_{A}(x)$ and $F_{A}(x)$, so $0^{-} \leq \sup T_{A}(x)+\sup I_{A}(x)+\sup F_{A}(x) \leq 3^{+}$.
Definition 4 ([75]). Let $X$ be a space of points or objects, with a generic element in $X$ denoted by $x$. A NS $A$ in $X$ is characterized by $T_{A}(x), I_{A}(x)$ and $F_{A}(x)$, which are singleton subintervals or subsets in the real standard $[0,1]$; that is $T_{A}(x): X \rightarrow[0,1], I_{A}(x): X \rightarrow$ $[0,1]$ and $F_{A}(x): X \rightarrow[0,1]$. Then, a simplification of NS $A$ is denoted by

$A=\left\{\left\langle x, T_{A}(x), I_{A}(x), F_{A}(x)\right\rangle \mid x \in X\right\}$,

which is a subclass of NS called a SNS.

Definition 5 ([67]). An INS $A$ in $X$ is characterized by a truthmembership function $T_{A}(x)$, an indeterminacy-membership function $I_{A}(x)$ and a falsity-membership function $F_{A}(x)$. For each point $x$ in $X$, it is true that

$T_{A}(x)=\left[\inf T_{A}(x), \sup T_{A}(x)\right], \quad I_{A}(x)=\left[\inf I_{A}(x), \sup I_{A}(x)\right]$,

$F_{A}(x)=\left[\inf F_{A}(x), \sup F_{A}(x)\right] \in[0,1]$

and $0 \leq \sup T_{A}+\sup I_{A}+\sup F_{A} \leq 3, x \in X$. Only the subunitary interval of $[0,1]$ is considered, and it is a subclass of NS. Therefore, all INSs are clearly NSs.

Definition 6. Let two INSs $A=\left\langle\left[\inf T_{A}, \sup T_{A}\right],\left[\inf I_{A}, \sup I_{A}\right]\right.$, $\left.\left[\inf F_{A}, \sup F_{A}\right]\right\rangle$ and $A=\left\langle\left[\inf T_{B}, \sup T_{B}\right],\left[\inf I_{B}, \sup I_{B}\right],\left[\inf F_{B}\right.\right.$, $\left.\left.\sup F_{B}\right]\right\rangle$, and $\lambda>0$. The INS operations are defined below [67].

(1) $A \oplus B=\left\langle\left[\inf T_{A}+\inf T_{B}-\inf T_{A} \cdot \inf T_{B}, \sup T_{A}\right.\right.$

$\left.+\sup T_{B}-\sup T_{A} \cdot \sup T_{B}\right]$,

$\left[\inf I_{A} \cdot \inf I_{B}, \sup I_{A} \cdot \sup I_{B}\right]$,

$\left.\left[\inf F_{A} \cdot \inf F_{B}, \sup F_{A} \cdot \sup F_{B}\right]\right\rangle$;

(2) $A \otimes B=\left\langle\left[\inf T_{A} \cdot \inf T_{B}, \sup T_{A} \cdot \sup T_{B}\right]\right.$,

$\left[\inf I_{A}+\inf I_{B}-\inf T_{A} \cdot \inf I_{B}, \sup I_{A}\right.$

$\left.+\sup I_{B}-\sup I_{A} \cdot \sup I_{B}\right]$,

$\left[\inf F_{A}+\inf F_{B}-\inf F_{A} \cdot \inf F_{B}, \sup F_{A}\right.$

$\left.\left.+\sup F_{B}-\sup F_{A} \cdot \sup F_{B}\right]\right\rangle$;

(3) $\lambda \cdot A=\left\langle\left[1-\left(1-\inf T_{A}\right)^{\lambda}, 1-\left(1-\sup T_{A}\right)^{\lambda}\right]\right.$,

$\left.\left[\left(\inf I_{A}\right)^{\lambda},\left(\sup I_{A}\right)^{\lambda}\right],\left[\left(\inf F_{A}\right)^{\lambda},\left(\sup F_{A}\right)^{\lambda}\right]\right\rangle ;$

(4) $A^{\lambda}=\left\langle\left[\left(\inf T_{A}\right)^{\lambda},\left(\sup T_{A}\right)^{\lambda}\right],\left[\left(\inf I_{A}\right)^{\lambda},\left(\sup I_{A}\right)^{\lambda}\right]\right.$,

$\left.\left[\left(\inf F_{A}\right)^{\lambda},\left(\sup F_{A}\right)^{\lambda}\right]\right\rangle$.

\subsection{CINS and its operators}

CINS is described in this section in order to measure the uncertainty of cloud services and compare candidate services with tradeoffs between performance-costs and potential risks.

Definition 7. The comprehensive evaluation of a cloud service is characterized by CINS $A=\left\langle\alpha_{A} O_{A}, \beta_{A} U_{A}, \gamma_{A} R_{A}\right\rangle . P_{A}=\left[\inf O_{A}\right.$, sup $O_{A}$ ], which represents the evaluation interval value of the performance-cost ratio, equivalent to the truth-membership function of INS. $R_{A}=\left[\inf R_{A}, \sup R_{A}\right]$ represents the evaluation interval value of the potential risks, equivalent to the falsity-membership function of INS. $U_{A}=\left[\inf U_{A}\right.$, sup $U_{A}$ ] represents the evaluation interval value of the uncertainty of $P_{A}$ and $R_{A}$, equivalent to the indeterminacy-membership function of INS. A larger $P_{A}$ with a smaller $U_{A}$ and $R_{A}$ yields a better evaluation. $O_{A}, R_{A}, U_{A} \in[0,1]$, and $0 \leq \sup O_{A}+\sup U_{A}+\sup R_{A} \leq 3 . \alpha, \beta$ and $\gamma$ are the tradeoff coefficients representing the importance degrees of the performance-cost ratio, uncertainty and potential risks, respectively, which should reflect the user's application requirements in different time periods. $\alpha, \beta, \gamma \in[0,1]$. Obviously, CINS is also an INS. 


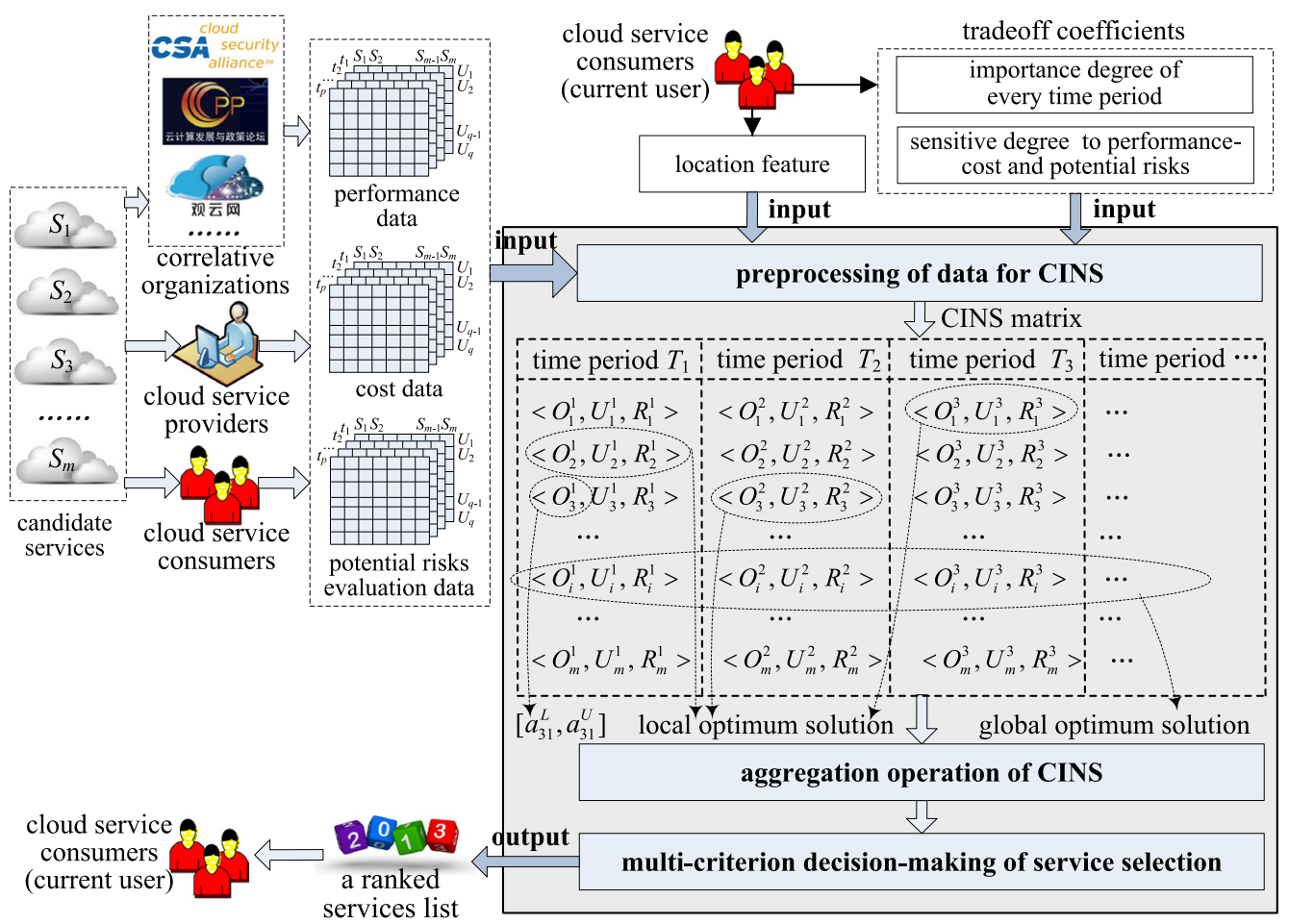

Fig. 1. Problem modeling.

Theorem 1. Let three CINSs $A=\left\langle\alpha_{A}\left[\inf O_{A}\right.\right.$, sup $\left.O_{A}\right], \beta_{A}\left[\inf U_{A}\right.$, $\left.\left.\sup U_{A}\right], \gamma_{A}\left[\inf R_{A}, \sup R_{A}\right]\right\rangle, B=\left\langle\alpha_{B}\left[\inf O_{B}, \sup P_{B}\right], \beta_{B}\left[\inf U_{B}\right.\right.$, $\left.\sup U_{B}\right], \gamma_{A}\left[\inf R_{B}\right.$, sup $\left.\left.R_{B}\right]\right\rangle$ and $C=\left\langle\alpha_{C}\left[\inf O_{C}, \sup P_{C}\right], \beta_{C}\left[\inf U_{C}\right.\right.$, $\sup U_{C}$ ], $\gamma_{C}\left[\inf R_{C}\right.$, sup $\left.\left.R_{C}\right]\right\rangle$, then the following equations are true.

(1) $A \oplus B=B \oplus A$;

(2) $A \otimes B=B \otimes A$;

(3) $\lambda \cdot(A \oplus B)=\lambda \cdot A \oplus \lambda \cdot B, \lambda>0$;

(4) $(A \otimes B)^{\lambda}=A^{\lambda} \otimes B^{\lambda}, \lambda>0$;

(5) $\lambda_{1} \cdot A \oplus \lambda_{2} \cdot A=\left(\lambda_{1}+\lambda_{2}\right) \otimes A, \lambda_{1}>0, \lambda_{2}>0$;

(6) $A^{\lambda_{1}} \otimes A^{\lambda_{2}}=A^{\left(\lambda_{1}+\lambda_{2}\right)}, \lambda_{1}>0, \lambda_{2}>0$;

(7) $(A \oplus B) \oplus C=A \oplus(B \oplus C)$;

(8) $(A \otimes B) \otimes C=A \otimes(B \otimes C)$.

Proof. Obviously, the proof of the above equations can be done according to Definition 6.

\section{Modeling problem}

\subsection{Problem definition}

For a list of $m$ candidate cloud services in the set $\left\{S_{1}, S_{2}, \ldots\right.$, $\left.S_{m-1}, S_{m}\right\}$ and a time series consisting of $p$ timeslots $\left\{t_{1}, t_{2}, \ldots\right.$, $\left.t_{p-1}, t_{p}\right\}$, assume that sufficient evaluation data exists about performance, cost and potential risk for every service in every timeslot. Full consideration of the geographic location feature of the current user allows these timeslots to be divided exactly into time periods in accordance with the time zone in which the current user lives. Based on an analysis of the application scenario requirements of current user, we can identify the importance degree of every time period, the sensitive degree of performance-cost ratio and the potential cloud service risks for the current user in every time period. Finally, the current user will receive a ranked list of recommended services $S_{\mathrm{r} 1} \succ S_{\mathrm{r} 2} \succ$ $\cdots \succ S_{r m-1} \succ S_{r m}$. The highest-ranked service in this list should demonstrate optimal performance-costs and potential risks in the specified time periods, and it should have a higher probability than lower-ranked services of being adopted by the current user.

In this problem, every time period becomes a decision criterion for evaluating the performance-costs and potential risks of cloud services. Thus, the time-aware trustworthy cloud service selection with tradeoffs between performance-costs and potential risks over multiple time periods can be formulated as a MCDM problem of creating a ranked services list, as shown in Fig. 1.

In Fig. 1, the sources of original evaluation data about cloud services mainly includes: (1) the performance data from continuous monitoring or continuous auditing, provided by service providers; (2) the continual evaluation data about performance, cost and potential risks, provided by correlative organizations, such as CSA, 3 CPP or yunzhiliang.net; (3) the feedback data about performance, cost and potential risks, provided by service consumers. By preprocessing these original data, the performance-cost evaluation and risks assessment of candidate services in every time period can be obtained. In time period $T_{i}$, the service with the optimal performance-cost and the minimal risk is the local optimum solution in the $i$ th criterion. The challenging task is to find the global optimum solution in all of time periods.

To address this MCDM problem, all original evaluation data about performance, cost and potential risks must first be preprocessed into the CINS matrix by integrating user's time zone information and tradeoff coefficients. After that, the CINS numbers for multiple time periods can be aggregated to compare the differences between candidate services in order to calculate the sort value of every service in ranked list of recommended services.

\subsection{Preprocessing of data for CINS}

The preprocessing of data for CINS consists of the following nine steps.

Step 1: Identifying the nearest neighbors for the current user. Based on the history performance evaluation data about cloud 
services, employ Pearson correlation coefficient to calculate the total similarity between the current user and other user by Eq. (3):

$\operatorname{sim}\left(u^{c}, u^{\circ}\right)=\frac{1}{\left|S^{\circ}\right|} \sum_{s_{i} \in S^{\circ}} \operatorname{sim}_{i}\left(u^{c}, u^{\circ}\right)$,

where $u^{c}$ represents the current user; $u^{\circ}$ represents other user who maybe a consumer, a service provider or a correlative organization; $S^{\circ}$ is a union set of evaluated services by $u^{c}$ and $u^{\circ} ; \operatorname{sim}_{i}\left(u^{c}, u^{\circ}\right)$ is the similarity between $u^{c}$ and $u^{\circ}$ obtained based on service $s_{i}$ as reference, which is calculated by Eq. (4):

$$
\operatorname{sim}_{i}\left(u^{c}, u^{\circ}\right)=\frac{\sum_{t \in T}\left(r_{t}^{c}-\overline{r^{c}}\right)\left(r_{t}^{\circ}-\overline{r^{\circ}}\right)}{\sqrt{\sum_{t \in T}\left(r_{t}^{c}-\overline{r^{c}}\right)^{2}} \sqrt{\sum_{t \in T}\left(r_{t}^{\circ}-\overline{r^{\circ}}\right)^{2}}} .
$$

Then, the set of nearest neighbors of $u^{c}$, noted as $N N$, can be selected by Eq. (5):

$N N=\left\{u^{i} \mid u^{i} \in S C, \operatorname{sim}\left(u^{c}, u^{i}\right) \geq \operatorname{sim}^{\text {th }}\right\}$

where SC represents the set of other consumers; $\operatorname{sim}^{\text {th }}$ is the threshold of user similarity.

Step 2: Collecting the evaluation data about performance, cost and potential risks of candidate services from nearest neighbors. Multiple evaluation indicators of performance and potential risks may exist for different types of cloud services. For example, the performance indicators for storage services include response time, accessing rate for storage interface, efficiency of data compression, whereas the performance indicators for computational intensive services include the number of concurrent users and the efficiency of instruction execution. Taking the performance evaluation as an example, the original evaluation matrix for a cloud storage service $s$ can be represented as follows:

$O(s)$

$$
=\left[\begin{array}{cccc}
\left(o_{11}^{1}, o_{11}^{2}, \ldots, o_{11}^{r}\right) & \left(o_{12}^{1}, o_{12}^{2}, \ldots, o_{12}^{r}\right) & \ldots & \left(o_{1 p}^{1}, o_{1 p}^{2}, \ldots, o_{1 p}^{r}\right) \\
\left(o_{21}^{1}, o_{21}^{2}, \ldots, o_{21}^{r}\right) & \left(o_{22}^{1}, o_{22}^{2}, \ldots, o_{22}^{r}\right) & \cdots & \left(o_{2 p}^{1}, o_{2 p}^{2}, \ldots, o_{2 p}^{r}\right) \\
\vdots & \vdots & \ldots & \vdots \\
\left(o_{q 1}^{1}, o_{q 1}^{2}, \ldots, o_{q 1}^{r}\right) & \left(o_{q 2}^{1}, o_{q 2}^{2}, \ldots, o_{q 2}^{r}\right) & \cdots & \left(o_{q p}^{1}, o_{q p}^{2}, \ldots, o_{q p}^{r}\right)
\end{array}\right],
$$

where $o_{i j}^{k}$ represents the evaluation value of the $k$ th performance indicator provided by the $i$ th user in timeslot $t_{j} ; p$ is the total number of timeslots; $q$ is the total number of users who have evaluated this service; $r$ is the total number of performance indicators. The potential risks of cloud services may associate multiple evaluation indicators. The multiple attributes evaluation matrix for potential risks can also be defined similarly by Eq. (6).

Step 3: Aggregating the multi-dimensional performance evaluations and risk evaluations into comprehensive evaluations with weighted arithmetic averaging operators. Because of the difference between gain-type indicators and loss-type indicators, the multidimensional evaluation data need to be normalized prior to aggregation operations. Gain-type indicators, such as accessing rate of storage interface and efficiency of data compression, can be normalized by Eq. (7):

$o_{i j}^{k}=\left(o_{i j}^{k}-\min _{i=1}^{q} o_{i j}^{k}\right) /\left(\max _{i=1}^{q} o_{i j}^{k}-\min _{i=1}^{q} o_{i j}^{k}\right)$.

Loss-type indicators, such as response time and availability risk, are normalized by Eq. (8):

$o_{i j}^{k}=\left(\max _{i=1}^{q} o_{i j}^{k}-o_{i j}^{k}\right) /\left(\max _{i=1}^{q} o_{i j}^{k}-\min _{i=1}^{q} o_{i j}^{k}\right)$.

The measurement unit of cost is usually inconsistent for different services; therefore, it should also be normalized with the same standard. In order to aggregate the multi-attributes evaluation data, let the weights matrix be $\omega=\left\{\omega_{1}, \omega_{2}, \ldots, \omega_{r}\right\} . \omega_{i}$ represents the weight the current user assigned to the ith attribute. The comprehensive performance evaluation of service $s$ provided by the $i$ th user in timeslot $t_{j}$ can be determined by Eq. (9):

$e_{i j}^{p}(s)=o_{i j} \cdot \omega^{T}=\left[\begin{array}{llll}o_{i j}^{1} & o_{i j}^{2} & \cdots & o_{i j}^{r}\end{array}\right]\left[\begin{array}{llll}\omega_{1} & \omega_{2} & \ldots & \omega_{r}\end{array}\right]^{T}$.

The comprehensive evaluation of potential risks can also be defined similarly by Eq. (9).

Step 4: Calculating the performance-cost ratio of every service in every timeslot. Let $e_{i j}^{P}$ and $e_{i j}^{C}$ be the comprehensive performance evaluation and normalized cost, respectively, in timeslot $t_{j}$ from the $i$ th user. Then, the performance-cost ratio is defined as $e_{i j}^{O}=$ $e_{i j}^{P} / e_{i j}^{C}$.

Step 5: Dividing timeslots into time periods on the basis of analyzing the user's time zone and application requirements. Let $d$ be the density coefficient of time period $T_{i}$, which represents the size of timeslots in $T_{i}$. Then the time period matrix is noted as follows:

$T=\left[\begin{array}{c}T_{1} \\ T_{2} \\ \vdots \\ T_{n}\end{array}\right]=\left[\begin{array}{cccc}t_{11} & t_{11} & \cdots & t_{1 d} \\ t_{21} & t_{22} & \cdots & t_{2 d} \\ \vdots & \vdots & \vdots & \vdots \\ t_{n 1} & t_{n 2} & \cdots & t_{n d}\end{array}\right]$

where $n$ is the number of time periods and $p=n \times d$. In practice, the density coefficient $d$ may be a variable, because the size of time period could be different.

Step 6: Transforming the single-value evaluation data with different timeslots into interval numbers in every time period by utilizing cloud model theory. The cloud model $[35,73]$ is a cognitive model realizing the bidirectional transformation between qualitative concept and quantitative data based on probability statistics and fuzzy set theory. It can effectively represent fuzziness, randomness and uncertain concepts, and it has been applied in many fields $[79,70,9]$. In this paper, we establish cloud models for performance-costs and potential risks based on evaluation data in order to identify their interval numbers in every time period. Let $E_{j, k}^{O}(s)=\left\{e_{j, d \times(k-1)+1}^{O}, e_{j, d \times(k-1)+2}^{O}, \ldots, e_{j, d \times k}^{O}\right\}$ be the single-value evaluation for performance-cost ratio of service $s$ provided by the $j$ th user in $T_{k}$. This data is viewed as cloud drops and sent into the reverse cloud generator (RCG). Then, the cloud model of performance-cost in $T_{i}$ can be obtained according to Eq. (11):

$$
\left\{\begin{array}{l}
E x_{i}^{O}=\frac{1}{d} \times \sum_{j=1}^{q} \sum_{k=1}^{d} e_{j, d *(k-1)+k}^{O} \\
E n_{i}^{O}=\sqrt{\frac{\pi}{2} \times \frac{1}{q \times d} \sum_{j=1}^{q} \sum_{k=1}^{d}\left|e_{j, d *(k-1)+k}^{O}-E x_{i}^{O}\right|} \\
H E_{i}^{O}=\sqrt{\left|\frac{1}{q \times d-1} \sum_{j=1}^{q} \sum_{k=1}^{d}\left(e_{j, d *(k-1)+k}^{O}-E x_{i}^{O}\right)^{2}-\left(E n_{i}^{O}\right)^{2}\right|},
\end{array}\right.
$$

where $q$ is the number of nearest neighbors and $d$ is the time period's density coefficient. The service's performance-cost ratio in $T_{i}$ is defined as $o_{i}$, described with an interval number as $o_{i}=$ $\left[o_{i}^{L}, o_{i}^{U}\right] . o_{i}^{L}$ and $o_{i}^{U}$ are calculated with Eq. (12):

$\left\{\begin{array}{l}o_{i}^{U}=E x_{i}^{O}+E n_{i}^{O}+H E_{i}^{O} \times \gamma \\ o_{i}^{L}=E x_{i}^{O}-E n_{i}^{O}-H E_{i}^{O} \times \gamma,\end{array}\right.$

where $\gamma$ is the influence coefficient of $H E$, suggested to remain in the interval range [0.1, 0.2] [39]. The cloud model for potential risks is established similarly, and the potential risks interval number for the service in $T_{i}$ defined as $r_{i}=\left[r_{i}^{L}, r_{i}^{U}\right]$ is obtained 
Table 1

Some typical values for tradeoff coefficients.

\begin{tabular}{|c|c|c|c|c|}
\hline Importance degree of current time period & Sensitive degree & $\alpha$ for performance-cost & $\beta$ for uncertainty & $\gamma$ for potential risks \\
\hline Very important & Sensitive to performance-cost & 1 & 0.8 & 0.8 \\
\hline Very important & Quite sensitive to performance-cost & 1 & 0.6 & 0.6 \\
\hline Important & Sensitive to performance-cost & 0.8 & 0.6 & 0.6 \\
\hline Important & Quite sensitive to performance-cost & 0.8 & 0.4 & 0.4 \\
\hline Very important & Sensitive to potential risks & 0.8 & 1 & 1 \\
\hline Very important & Quite sensitive to potential risks & 0.6 & 1 & 1 \\
\hline Important & Sensitive to potential risks & 0.6 & 0.8 & 0.8 \\
\hline Important & Quite sensitive to potential risks & 0.4 & 0.8 & 0.8 \\
\hline Not important & No preferences & 0.5 & 0.5 & 0.5 \\
\hline Pay no attention & No preferences & 0 & 0 & 0 \\
\hline
\end{tabular}

with Eq. (13):

$$
\left\{\begin{array}{l}
r_{i}^{L}=E x_{i}^{R}-E n_{i}^{R}-H E_{i}^{R} \times \gamma \\
r_{i}^{U}=E x_{i}^{R}+E n_{i}^{R}+H E_{i}^{R} \times \gamma .
\end{array}\right.
$$

Step 7: Calculating the uncertainty interval of performance-cost ratio and potential risks in $T_{i}$. Let $\lambda_{i}^{O}=\sqrt{\left(E n_{i}^{O}\right)^{2}+\left(H E_{i}^{O}\right)^{2}}$ and $\lambda_{i}^{R}=\sqrt{\left(E n_{i}^{R}\right)^{2}+\left(H E_{i}^{R}\right)^{2}}$ be the uncertainty of performance-cost ratio and the uncertainty of potential risks, respectively, in $T_{i}$. Then, the comprehensive uncertainty interval of $T_{i}$ is defined as Eq. (14):

$U_{i}=\left[u_{i}^{L}, u_{i}^{L}\right]=\left[\min \left\{\lambda_{i}^{O}, \lambda_{i}^{R}\right\}, \max \left\{\lambda_{i}^{O}, \lambda_{i}^{R}\right\}\right]$.

Then, the original evaluation data is noted with CINS as follows:

$$
\begin{aligned}
E & =\left(\begin{array}{c}
E_{1} \\
E_{2} \\
\vdots \\
E_{m}
\end{array}\right)=\left(\begin{array}{cccc}
e_{11} & e_{12} & \cdots & e_{1 n} \\
e_{21} & e_{22} & \cdots & e_{2 n} \\
\vdots & \vdots & \vdots & \vdots \\
e_{m 1} & e_{m 2} & \cdots & e_{m n}
\end{array}\right) \\
& =\left(\begin{array}{ccc}
\left\langle O_{11}, U_{11}, R_{11}\right\rangle & \cdots & \left\langle O_{1 n}, U_{1 n}, R_{1 n}\right\rangle \\
\vdots & \ddots & \vdots \\
\left\langle O_{m 1}, U_{m 1}, R_{m 1}\right\rangle & \cdots & \left\langle O_{m n}, U_{m n}, R_{m n}\right\rangle
\end{array}\right) .
\end{aligned}
$$

Step 8: Analyzing the user's application scenario requirements and identifying the tradeoff coefficients $\alpha, \beta$ and $\gamma$ for performance-cost, uncertainty and potential risks, respectively, in every time period. These coefficients should reflect the importance degree of every time period and the sensitive degrees of performance-cost ratio and potential risks for the cloud service. The time periods' tradeoff coefficient is defined as

$F=\left(\begin{array}{c}F_{1} \\ F_{2} \\ \vdots \\ F_{n}\end{array}\right)=\left(\begin{array}{ccc}\alpha_{1}, & \beta_{1}, & \gamma_{1} \\ \alpha_{2}, & \beta_{2}, & \gamma_{2} \\ \vdots & \vdots & \vdots \\ \alpha_{n}, & \beta_{n}, & \gamma_{n}\end{array}\right)$

where $F_{i}$ represents the tradeoff coefficient of $T_{i}$ assigned by the current user. Table 1 shows some typical values for tradeoff coefficients.

In Table $1, \alpha, \beta, \gamma \in[0,1]$, the current user can determine their values according to the importance of every time period and tradeoffs between performance-cost and potential risks. The fundamental principles of determining the tradeoff coefficients mainly include: (1) if the current time period is very important or has a greater importance than other time periods, $0.6 \leq \alpha, \beta, \gamma \leq$ 1 ; (2) if the current time period is important, $0.5 \leq \alpha, \beta, \gamma \leq 0.8$; (3) if the current time period is not important, $0.1 \leq \alpha, \beta, \gamma \leq$ 0.5 ; (4) if user pays no attention to the current time period, $\alpha=$ $0, \beta=0, \gamma=0$; (5) if user is sensitive to performance-cost, $(\alpha-\beta) \geq 0.2$ and $(\alpha-\gamma) \geq 0.2$; (6) if user is quite sensitive to performance-cost, $(\alpha-\beta) \geq 0.4$ and $(\alpha-\gamma) \geq 0.4$; (7) if user is sensitive to potential risks, $(\beta-\alpha) \geq 0.2$ and $(\gamma-\alpha) \geq 0.2$; (8) if user is quite sensitive to potential risks, $(\beta-\alpha) \geq 0.4$ and $(\gamma-\alpha) \geq 0.4$.

Step 9: Assembling preprocessed evaluation data for candidate services in all time periods using CINS theory. According to the tradeoff coefficient $F$, a matrix is defined as follows:

$Y=\left(\begin{array}{cccccc}F_{1} & 0 & \cdots & \cdots & \cdots & 0 \\ 0 & F_{2} & 0 & \cdots & \cdots & 0 \\ 0 & 0 & F_{3} & 0 & \cdots & 0 \\ 0 & \cdots & \cdots & \cdots & \cdots & 0 \\ 0 & \cdots & \cdots & \cdots & \cdots & F_{n}\end{array}\right)$

$$
=\left(\begin{array}{cccccc}
\left(\alpha_{1}, \beta_{1}, \gamma_{1}\right) & 0 & \cdots & \cdots & \cdots & 0 \\
0 & \left(\alpha_{2}, \beta_{2}, \gamma_{2}\right) & 0 & \cdots & \cdots & 0 \\
0 & 0 & \left(\alpha_{3}, \beta_{3}, \gamma_{3}\right) & 0 & \cdots & 0 \\
0 & \cdots & \cdots & \cdots & \cdots & 0 \\
0 & \cdots & \cdots & \cdots & \cdots & \left(\alpha_{n}, \beta_{n}, \gamma_{n}\right)
\end{array}\right) .
$$

Then, the comprehensive evaluation decision matrix of candidate services is given as in Box I, where $v_{i j}$ is a CINS representing the total evaluation of the $i$ th service in $T_{i}$.

The most trustworthy service should have the optimal evaluation in $V$. In order to pick out the optimal candidate from $m$ services in $n$ time periods, the aggregation operators and entropy weight measure method for CINS will be designed and proved theoretically.

\section{Proposed CINS aggregation methodology}

\subsection{Aggregation operators for CINS}

Definition 8. Let $A_{i}=\left\langle\alpha_{A_{i}} O_{A_{i}}, \beta_{A_{i}} U_{A_{i}}, \gamma_{A_{i}} R_{A_{i}}\right\rangle(i=1,2, \ldots, n)$ be a collection of CINSs. The CINSs' aggregation operators are defined as CINSWA : CINS $^{n} \rightarrow$ CINS,

$$
\begin{aligned}
& \operatorname{CINSWA}_{w}\left(A_{1}, A_{2}, \ldots, A_{n}\right) \\
& \quad=w_{1} \cdot A_{1} \oplus w_{2} \cdot A_{2} \oplus \cdots \oplus w_{n} \cdot A_{n}=\sum_{i=1}^{n} w_{i} A_{i},
\end{aligned}
$$

where $W=\left(w_{1}, w_{2}, \ldots, w_{n}\right)$ is weight vector of $A_{i}(i=1,2$, $\ldots, n), w_{i} \geq 0(i=1,2, \ldots, n)$ and $\sum_{i=1}^{n} w_{i}=1$. CINSWA represents the CINS weighted averaging operator for the $n$ dimension.

Theorem 2. Let $A_{i}=\left\langle\alpha_{A_{i}} O_{A_{i}}, \beta_{A_{i}} U_{A_{i}}, \gamma_{A_{i}} R_{A_{i}}\right\rangle(i=1,2, \ldots, n)$ be a collection of CINSs, and let $W=\left(w_{1}, w_{2}, \ldots, w_{n}\right)$ be the weight vector of $A_{i}(i=1,2, \ldots, n)$ with $w_{i} \geq 0(i=1,2, \ldots, n)$ and $\sum_{i=1}^{n} w_{i}=1$. Then, their aggregated result using CINSWA operator is also a CINS, and

$$
\begin{aligned}
& \operatorname{CINSWA}_{w}\left(A_{1}, A_{2}, \ldots, A_{n}\right) \\
& \quad=\left\langle\left[1-\prod_{i=1}^{n}\left(1-\alpha_{A_{i}} \inf O_{A_{i}}\right)^{w_{i}}, 1-\prod_{i=1}^{n}\left(1-\alpha_{A_{i}} \sup O_{A_{i}}\right)^{w_{i}}\right],\right.
\end{aligned}
$$




$$
\begin{aligned}
V & =\left(\begin{array}{c}
V_{1} \\
\vdots \\
V_{m}
\end{array}\right)=\left(\begin{array}{ccc}
v_{11} & \cdots & v_{1 n} \\
\vdots & \ddots & \vdots \\
v_{m 1} & \cdots & v_{m n}
\end{array}\right) \\
& =E \otimes Y=\left(\begin{array}{ccc}
\left\langle\alpha_{1} O_{11}, \beta_{1} U_{11}, \gamma_{1} R_{11}\right\rangle & \cdots & \left\langle\alpha_{n} O_{1 n}, \beta_{n} U_{1 n}, \gamma_{n} R_{1 n}\right\rangle \\
\vdots & \ddots & \vdots \\
\left\langle\alpha_{1} O_{m 1}, \beta_{1} U_{m 1}, \gamma_{1}, R_{m 1}\right\rangle & \ldots & \left\langle\alpha_{n} O_{m n}, \beta_{n} U_{m n}, \gamma_{n} R_{m n}\right\rangle
\end{array}\right) \\
& =\left(\begin{array}{cccc}
\left\langle\alpha_{1}\left[o_{11}^{L}, o_{11}^{U}\right], \beta_{1}\left[u_{11}^{L}, u_{11}^{U}\right],\right. & \left.\gamma_{1}\left[r_{11}^{L}, r_{11}^{U}\right]\right\rangle & \ldots & \left\langle\alpha_{n}\left[o_{1 n}^{L}, o_{1 n}^{U}\right], \beta_{n}\left[u_{1 n}^{L}, u_{1 n}^{U}\right], \gamma_{n}\left[r_{1 n}^{L}, r_{1 n}^{U}\right]\right\rangle \\
\vdots & \vdots \\
\left\langle\alpha_{1}\left[o_{m 1}^{L}, o_{m 1}^{U}\right], \beta_{1}\left[u_{m 1}^{L}, u_{m 1}^{U}\right], \gamma_{1}\left[r_{m 1}^{L}, r_{m 1}^{U}\right]\right\rangle & \cdots & \left\langle\alpha_{n}\left[o_{m n}^{L}, o_{m n}^{U}\right], \beta_{n}\left[u_{m n}^{L}, u_{m n}^{U}\right], \gamma_{n}\left[r_{m n}^{L}, r_{m n}^{U}\right]\right\rangle
\end{array}\right),
\end{aligned}
$$

Box I.

$$
\begin{aligned}
& {\left[\prod_{i=1}^{n}\left(\beta_{A_{i}} \inf U_{A_{i}}\right)^{w_{i}}, \prod_{i=1}^{n}\left(\beta_{A_{i}} \sup U_{A_{i}}\right)^{w_{i}}\right],} \\
& \left.\left[\prod_{i=1}^{n}\left(\gamma_{A_{i}} \inf R_{A_{i}}\right)^{w_{i}}, \prod_{i=1}^{n}\left(\gamma_{A_{i}} \sup R_{A_{i}}\right)^{w_{i}}\right]\right\rangle,
\end{aligned}
$$

where $w_{i} \in[0,1]$ and $\sum_{i=1}^{n} w_{i}=1$.

Proof. Eq. (18) can be proven by means of mathematical induction.

When $n=2$, then $\operatorname{CINSWA}_{w}\left(A_{1}, A_{2}\right)=w_{1} \cdot A_{1} \oplus w_{2} \cdot A_{2}$. According to Definition $6, w_{1} \cdot A_{1}=\left\langle\left[1-\left(1-\alpha_{A_{1}} \text { inf } O_{A_{1}}\right)^{w_{1}}, 1-(1-\right.\right.$ $\left.\left.\alpha_{A_{1}} \sup O_{A_{1}}\right)^{w_{1}}\right],\left[\left(\beta_{A_{1}} \inf U_{A_{1}}\right)^{w_{1}},\left(\beta_{A_{1}} \sup U_{A_{1}}\right)^{w_{1}}\right]$,

$\left.\left[\left(\gamma_{A_{1}} \inf R_{A_{1}}\right)^{w_{1}},\left(\gamma_{A_{1}} \sup R_{A_{1}}\right)^{w_{1}}\right]\right\rangle$ and $w_{2} \cdot A_{2}=\langle[1-(1-$ $\left.\left.\alpha_{A_{2}} \inf O_{A_{2}}\right)^{w_{2}}, 1-\left(1-\alpha_{A_{2}} \sup O_{A_{2}}\right)^{w_{2}}\right],\left[\left(\beta_{A_{2}} \inf U_{A_{2}}\right)^{w_{2}}\right.$, $\left.\left.\left(\beta_{A_{2}} \sup U_{A_{2}}\right)^{w_{2}}\right],\left[\left(\gamma_{A_{2}} \inf R_{A_{2}}\right)^{w_{2}},\left(\gamma_{A_{2}} \sup R_{A_{2}}\right)^{w_{2}}\right]\right\rangle$. Then,

$$
\begin{aligned}
w_{1} \cdot & A_{1} \oplus w_{2} \cdot A_{2} \\
= & \left\langle\left[\left(1-\left(1-\alpha_{A_{1}} \inf O_{A_{1}}\right)^{w_{1}}\right)+\left(1-\left(1-\alpha_{A_{2}} \inf O_{A_{2}}\right)^{w_{2}}\right)\right.\right. \\
& -\left(1-\left(1-\alpha_{A_{1}} \inf O_{A_{1}}\right)^{w_{1}}\right) \times\left(1-\left(1-\alpha_{A_{2}} \inf O_{A_{2}}\right)^{w_{2}}\right), \\
& \left(1-\left(1-\alpha_{A_{1}} \sup O_{A_{1}}\right)^{w_{1}}\right)+\left(1-\left(1-\alpha_{A_{2}} \sup O_{A_{2}}\right)^{w_{2}}\right) \\
& \left.-\left(1-\left(1-\alpha_{A_{1}} \sup O_{A_{1}}\right)^{w_{1}}\right) \times\left(1-\left(1-\alpha_{A_{2}} \sup O_{A_{2}}\right)^{w_{2}}\right)\right], \\
& {\left[\left(\beta_{A_{1}} \sup U_{A_{1}}\right)^{w_{1}} \cdot \times\left(\beta_{A_{2}} \inf U_{A_{2}}\right)^{w_{2}},\left(\beta_{A_{1}} \sup U_{A_{1}}\right)^{w_{1}}\right.} \\
& \left.\times\left(\beta_{A_{2}} \sup U_{A_{2}}\right)^{w_{2}}\right], \\
& {\left[\left(\gamma_{A_{1}} \inf R_{A_{1}}\right)^{w_{1}} \times\left(\gamma_{A_{2}} \inf R_{A_{2}}\right)^{w_{2}},\left(\gamma_{A_{1}} \sup R_{A_{1}}\right)^{w_{1}}\right.} \\
& \left.\left.\times\left(\gamma_{A_{2}} \sup R_{A_{2}}\right)^{w_{2}}\right]\right\rangle \\
= & \left\langle\left[1-\prod_{i=1}^{2}\left(1-\alpha_{A_{i}} \inf O_{A_{i}}\right)^{w_{i}}, 1-\prod_{i=1}^{2}\left(1-\alpha_{A_{i}} \sup O_{A_{i}}\right)^{w_{i}}\right],\right. \\
& {\left[\prod_{i=1}^{2}\left(\beta_{A_{i}} \inf U_{A_{i}}\right)^{w_{i}}, \prod_{i=1}^{2}\left(\beta_{A_{i}} \sup U_{A_{i}}\right)^{w_{i}}\right], } \\
& {\left.\left[\prod_{i=1}^{2}\left(\gamma_{A_{i}} \inf R_{A_{i}}\right)^{w_{i}}, \prod_{i=1}^{2}\left(\gamma_{A_{i}} \sup R_{A_{i}}\right)^{w_{i}}\right]\right\rangle . }
\end{aligned}
$$

Thus, the equation holds.

Assume that the equation holds when $n=k$. Then when $n=$ $k+1$,

$$
\begin{aligned}
\operatorname{CSINNWA}_{w}\left(A_{1}, A_{2}, \ldots, A_{k}, A_{k+1}\right) \\
=\left(w_{1} \cdot A_{1} \oplus w_{2} \cdot A_{2} \oplus \cdots \oplus w_{k} \cdot A_{k}\right) \oplus w_{k+1} \cdot A_{k+1} \\
=\left\langle\left[1-\prod_{i=1}^{k}\left(1-\alpha_{A_{i}} \inf O_{A_{i}}\right)^{w_{i}}, 1-\prod_{i=1}^{k}\left(1-\alpha_{A_{i}} \sup O_{A_{i}}\right)^{w_{i}}\right],\right. \\
\quad \times\left[\prod_{i=1}^{k}\left(\beta_{A_{i}} \inf U_{A_{i}}\right)^{w_{i}}, \prod_{i=1}^{k}\left(\beta_{A_{i}} \sup U_{A_{i}}\right)^{w_{i}}\right],
\end{aligned}
$$

$$
\begin{aligned}
& \left.\times\left[\prod_{i=1}^{k}\left(\gamma_{A_{i}} \inf R_{A_{i}}\right)^{w_{i}}, \prod_{i=1}^{k}\left(\gamma_{A_{i}} \sup R_{A_{i}}\right)^{w_{i}}\right]\right\rangle \\
& \oplus w_{k+1} A_{k+1}
\end{aligned}
$$

and

$$
\begin{aligned}
w_{k+1} \cdot A_{k+1}= & \left\langle\left[ 1-\left(1-\alpha_{A_{k+1}} \inf O_{A_{k+1}}\right)^{w_{k+1}},\right.\right. \\
& \left.1-\left(1-\alpha_{A_{k+1}} \sup O_{A_{k+1}}\right)^{w_{k+1}}\right], \\
& {\left[\left(\beta_{A_{k+1}} \inf U_{A_{k+1}}\right)^{w_{k+1}},\left(\beta_{A_{k+1}} \sup U_{A_{k+1}}\right)^{w_{k+1}}\right], } \\
& {\left.\left[\left(\gamma_{A_{k+1}} \inf R_{A_{k+1}}\right)^{w_{k+1}},\left(\gamma_{A_{k+1}} \sup R_{A_{k+1}}\right)^{w_{k+1}}\right]\right\rangle . }
\end{aligned}
$$

Then, according to Definition 6,

$$
\begin{aligned}
& \operatorname{CSINNWA}_{w}\left(A_{1}, A_{2}, \ldots, A_{k}, A_{k+1}\right) \\
& =\left\langle\left[\left(1-\prod_{i=1}^{k}\left(1-\alpha_{A_{i}} \inf O_{A_{i}}\right)^{w_{i}}\right)\right.\right. \\
& +\left(1-\left(1-\alpha_{A_{k+1}} \inf O_{A_{k+1}}\right)^{w_{k+1}}\right) \\
& -\left(1-\prod_{i=1}^{k}\left(1-\alpha_{A_{i}} \inf O_{A_{i}}\right)^{w_{i}}\right) \\
& \times\left(1-\left(1-\alpha_{A_{k+1}} \inf O_{A_{k+1}}\right)^{w_{k+1}}\right), \\
& \left(1-\prod_{i=1}^{k}\left(1-\alpha_{A_{i}} \sup O_{A_{i}}\right)^{w_{i}}\right) \\
& \times\left(1-\left(1-\alpha_{A_{k+1}} \sup O_{A_{k+1}}\right)^{w_{k+1}}\right) \\
& -\left(1-\prod_{i=1}^{k}\left(1-\alpha_{A_{i}} \sup O_{A_{i}}\right)^{w_{i}}\right) \\
& \left.\times\left(1-\left(1-\alpha_{A_{k+1}} \sup O_{A_{k+1}}\right)^{w_{k+1}}\right)\right] \text {, } \\
& {\left[\prod_{i=1}^{k}\left(\beta_{A_{i}} \inf U_{A_{i}}\right)^{w_{i}} \times\left(\left(\beta_{A_{k+1}} \inf U_{A_{k+1}}\right)^{w_{k+1}}\right)\right. \text {, }} \\
& \left.\prod_{i=1}^{k}\left(\beta_{A_{i}} \sup U_{A_{i}}\right)^{w_{i}} \times\left(\left(\beta_{A_{k+1}} \sup U_{A_{k+1}}\right)^{w_{k+1}}\right)\right] \text {, } \\
& {\left[\prod_{i=1}^{k}\left(\gamma_{A_{i}} \inf R_{A_{i}}\right)^{w_{i}} \times\left(\left(\gamma_{A_{k+1}} \inf R_{A_{k+1}}\right)^{w_{k+1}}\right),\right.} \\
& \left.\left.\prod_{i=1}^{k}\left(\gamma_{A_{i}} \sup R_{A_{i}}\right)^{w_{i}} \times\left(\left(\gamma_{A_{k+1}} \sup R_{A_{k+1}}\right)^{w_{k+1}}\right)\right]\right\rangle \\
& =\left\langle\left[1-\prod_{i=1}^{k+1}\left(1-\alpha_{A_{i}} \inf O_{A_{i}}\right)^{w_{i}}, 1-\prod_{i=1}^{k+1}\left(1-\alpha_{A_{i}} \sup O_{A_{i}}\right)^{w_{i}}\right]\right. \text {, } \\
& {\left[\prod_{i=1}^{k+1}\left(\beta_{A_{i}} \inf U_{A_{i}}\right)^{w_{i}}, \prod_{i=1}^{k+1}\left(\beta_{A_{i}} \sup U_{A_{i}}\right)^{w_{i}}\right] \text {, }}
\end{aligned}
$$




$$
\left.\left[\prod_{i=1}^{k+1}\left(\gamma_{A_{i}} \inf R_{A_{i}}\right)^{w_{i}}, \prod_{i=1}^{k+1}\left(\gamma_{A_{i}} \sup R_{A_{i}}\right)^{w_{i}}\right]\right\rangle .
$$

Considering the above results, Eq. (18) holds for any $n$. This completes the proof.

The CINSWA operator has the following properties:

(1) Idempotency: Let $A_{i}(i=1,2, \ldots, n)$ be a collection of CINSs If all $A_{i}(i=1,2, \ldots, n)$ are equal, that is, $A_{i}=A$, for all $i \in\{1,2, \ldots, n\}$, then $\operatorname{CINSWA}_{w}\left(A_{1}, A_{2}, \ldots, A_{n}\right)=A$.

(2) Boundedness: Assume $A_{i}(i=1,2, \ldots, n)$ is a collection of CINSs, and $A^{U}$ and $A^{L}$ are defined as follows:

$$
\begin{aligned}
& A^{U}=\left\langle\max _{i=1}^{n}\left\{\alpha_{A_{i}} O_{A_{i}}(x)\right\}, \min _{i=1}^{n}\left\{\beta_{A_{i}} U_{A_{i}}(x)\right\}, \min _{i=1}^{n}\left\{\gamma_{A_{i}} R_{A_{i}}(x)\right\}\right\rangle \\
& \max _{i=1}^{n}\left\{\alpha_{A_{i}}\left[\inf O_{A_{i}}(x), \sup O_{A_{i}}(x)\right]\right\},
\end{aligned}
$$

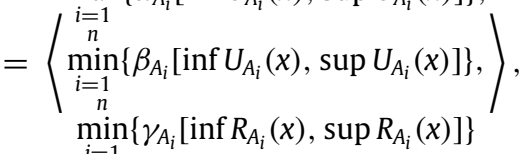

$$
\begin{aligned}
& A^{L}=\left\langle\min _{i=1}^{n}\left\{\alpha_{A_{i}} O_{A_{i}}(x)\right\}, \max _{i=1}^{n}\left\{\beta_{A_{i}} U_{A_{i}}(x)\right\}, \max _{i=1}^{n}\left\{\gamma_{A_{i}} R_{A_{i}}(x)\right\}\right\rangle \\
& =\left\langle\begin{array}{c}
\min _{i=1}^{n}\left\{\alpha_{A_{i}}\left[\inf O_{A_{i}}(x), \sup O_{A_{i}}(x)\right]\right\}, \\
\max _{i=1}^{n}\left\{\beta_{A_{i}}\left[\inf U_{A_{i}}(x), \sup U_{A_{i}}(x)\right]\right\}, \\
\max _{i=1}^{n}\left\{\gamma_{A_{i}}\left[\inf R_{A_{i}}(x), \sup R_{A_{i}}(x)\right]\right\}
\end{array}\right\rangle .
\end{aligned}
$$

The comparison operation between interval numbers can be executed using Eq. (1). Based on the possibility degree matrix, the sort value of each interval number can be calculated by Eq. (2). For all $i \in\{1,2, \ldots, n\}, A^{L} \in \operatorname{CINSWA}_{w}\left(A_{1}, A_{2}, \ldots, A_{n}\right)$ $\in A^{U}$.

(3) Monotonicity: Assuming $A_{i}(i=1,2, \ldots, n)$ is a collection of CINSs, if $A_{i} \subseteq A_{i}^{*}$ and $i \in\{1,2, \ldots, n\}$, then $\operatorname{CINSWA}_{w}\left(A_{1}, A_{2}, \ldots, A_{n}\right) \in \operatorname{CINSWA}_{w}\left(A_{1}^{*}, A_{2}^{*}, \ldots, A_{n}^{*}\right)$.

\subsection{Entropy weight measure for CINS}

In information theory, entropy is a measure for calculating the uncertainty associated with a random variable. Therefore, it is reasonable to utilize entropy as a vehicle to obtain objective weight [74]. According to entropy theory, if a decision criterion in a MCDM problem provides more uncertainty than others, it may be paid more attention. The entropy weight measure for CINSs is defined based on the axiomatic definition of the entropy measure for single-valued neutrosophic sets [41], in order to calculate the weights of time periods.

Definition 9. The entropy on $\operatorname{CINS}(X)$ is a real function $E$ : $\operatorname{CINS}(X)$ $\rightarrow[0,1]$ if $E$ satisfies the following properties:

(P1) $E(A)=0$ if the cloud service associated with $A$ is totally trustworthy;

(P2) $E(A)=1$ if the cloud service associated with $A$ is totally untrusted.

Definition 10. Let $A$ be a CINS in the universal discourse $X=$ $\left\{x_{1}, x_{2}, \ldots, x_{n}\right\} . E(A)$ is a measure such that

$E(A)=1-d\left(A, A^{+}\right)$, where $d\left(A, A^{+}\right)$refers to the distance measure between CINS $A$ and positive ideal CINS $A^{+} . A^{+}=\left\langle\left[\inf P_{A^{+}}, \sup P_{A^{+}}\right]\right.$, [inf $\left.U_{A^{+}}, \sup U_{A^{+}}\right]$, $\left.\left[\inf R_{A^{+}}, \sup R_{A^{+}}\right]\right\rangle=\langle[1,1],[0,0],[0,0]\rangle$.

Definition 11 ([76]). Let $A$ and $B$ be two CINSs in the universal discourse $X=\left\{x_{1}, x_{2}, \ldots, x_{m}\right\}$. Then, the Euclidean distance between them is calculated with Eq. (20):

$$
\begin{aligned}
d\left(A, A^{+}\right) & \\
= & \frac{1}{6}\left[\left(\alpha_{A} \inf O_{A\left(x_{i}\right)}-\inf O_{A^{+}}\right)^{2}+\left(\alpha_{A} \sup O_{A\left(x_{i}\right)}-\sup O_{A^{+}}\right)^{2}\right. \\
& +\left(\beta_{A} \inf U_{A\left(x_{i}\right)}-\inf U_{A^{+}}\right)^{2}+\left(\beta_{A} \sup U_{A\left(x_{i}\right)}-\sup U_{A^{+}}\right)^{2} \\
& \left.+\left(\gamma_{A} \inf R_{A\left(x_{i}\right)}-\inf R_{A^{+}}\right)^{2}+\left(\gamma_{A} \sup R_{A\left(x_{i}\right)}-\sup R_{A^{+}}\right)^{2}\right]^{1 / 2}
\end{aligned}
$$

Theorem 3. The proposed measure $E(A)$ satisfies all the axioms given in Definition 9.

Proof. Let $A=\left\langle\alpha_{A}\left[\inf O_{A}, \sup O_{A}\right], \beta_{A}\left[\inf U_{A}, \sup U_{A}\right], \gamma_{A}\left[\inf R_{A}\right.\right.$, $\left.\left.\sup R_{A}\right]\right\rangle$ be a CINS, and $A^{+}=\left\langle\left[\inf P_{A^{+}}, \sup P_{A^{+}}\right]\right.$, [inf $\left.U_{A^{+}}, \sup U_{A^{+}}\right]$, $\left.\left[\inf R_{A^{+}}, \sup R_{A^{+}}\right]\right\rangle=\langle[1,1],[0,0],[0,0]\rangle$.

(P1) If the cloud service associated with $A$ is totally trustworthy, $\alpha_{A} \inf O_{A}=\alpha_{A} \sup O_{A}=1, \beta_{A} \inf U_{A}=\beta_{A} \sup U_{A}=0$, $\gamma_{A} \inf R_{A}=\gamma_{A} \sup R_{A}=0$. Then, $A$ is a positive ideal CINS, $A=\langle[1,1],[0,0],[0,0]\rangle$. Therefore, $E(A)=0$, and (P1) in Definition 9 holds.

(P2) If the cloud service associated with $A$ is totally untrusted, $\alpha_{A} \inf O_{A}=\alpha_{A} \sup O_{A}=0, \beta_{A} \inf U_{A}=\beta_{A} \sup U_{A}=1$, $\gamma_{A}$ inf $R_{A}=\gamma_{A}$ sup $R_{A}=1$. Then, $A$ is a negative ideal CINS, $A=\langle[0,0],[1,1],[1,1]\rangle$. Therefore, $E(A)=1$, and $(\mathrm{P} 2)$ in Definition 9 holds.

Definition 12. Let CINS $v_{i j}$ be the comprehensive evaluation of the $i$ th service in the $j$ th time period in $V$ defined by Eq. (16). The entropy of the $j$ th time period is obtained by the average entropy distance between any two $v_{i j}$ in this time period, which is noted as follows:

$E\left(T_{j}\right)=1-\frac{2}{m(m-1)} \sum_{i=1}^{m} \sum_{k=i+1}^{m}\left|E\left(v_{i j}\right)-E\left(v_{k j}\right)\right|$,

where $E\left(v_{i j}\right)$ is calculated by Eq. (19).

According to these theories, an entropy weight measure is established to determine the weight of every time period under the CINS environment:

$W\left(T_{i}\right)=\left(1-E\left(T_{i}\right)\right) /\left(n-\sum_{i=1}^{n} E\left(T_{i}\right)\right)$.

Property 1. The proposed weight measure satisfies the following properties:

(P1) $W\left(T_{i}\right) \in[0,1]$;

(P2) $\sum_{i=1}^{n} W\left(T_{i}\right)=1$.

Proof. (P1) Let $W=\left(W\left(T_{1}\right), W\left(T_{2}\right), \ldots, W\left(T_{n}\right)\right)$ be an entropy weight vector calculated according to Eq. (22). According to Theorem 3, the entropy value of CINS lies between 0 and 1 , that is, $E\left(v_{i j}\right) \in[0,1]$; therefore, $\left|E\left(v_{i j}\right)-E\left(v_{k j}\right)\right| \in[0,1]$, the average entropy distance between any two $v_{i j}$, namely,

$$
\frac{2}{m(m-1)} \sum_{i=1}^{m} \sum_{k=i+1}^{m}\left|E\left(v_{i j}\right)-E\left(v_{k j}\right)\right| \in[0,1],
$$


and $1-E\left(T_{i}\right) \in[0,1]$. Additionally,

$\left(1-E\left(T_{i}\right)\right)+\left(n-1+\sum_{i=1, i \neq j}^{n} E\left(T_{i}\right)\right)=n-\sum_{i=1}^{n} E\left(T_{i}\right) \geq 0$

and

$\left(n-1+\sum_{i=1, i \neq j}^{n} E\left(T_{i}\right)\right) \geq 0$

hold, which means that

$n-\sum_{i=1}^{n} E\left(T_{i}\right) \geq\left(1-E\left(T_{i}\right)\right)$

is true. Based on these conclusions, it is possible to obtain

$W\left(T_{i}\right)=\frac{1-E\left(T_{i}\right)}{n-\sum_{i=1}^{n} E\left(T_{i}\right)} \in[0,1]$.

(P2) It is obvious that

$$
\begin{aligned}
\sum_{i=1}^{n} W\left(T_{i}\right) & =\sum_{i=1}^{n} \frac{1-E\left(T_{i}\right)}{n-\sum_{j=1}^{n} E\left(T_{j}\right)} \\
& =\frac{\sum_{i=1}^{n}\left(1-E\left(T_{i}\right)\right)}{n-\sum_{j=1}^{n} E\left(T_{j}\right)}=\frac{n-\sum_{i=1}^{n} E\left(T_{i}\right)}{n-\sum_{j=1}^{n} E\left(T_{j}\right)}=1 .
\end{aligned}
$$

Therefore, Property 1 holds.

In particular, Table 1 states that if the current users pay no attention to the $i$ th time period, then $\alpha_{i}=\beta_{i}=\gamma_{i}=0$. In this case, every $v_{i j}$ in the $i$ th time period will be substituted with the negative ideal CINS, which will ensure $W\left(T_{i}\right)=0$ in the entropy weight measure.

\section{MCDM procedure for time-aware trustworthy service selec- tion}

Assume there are $m$ cloud services $S=\left\{S_{1}, S_{2}, \ldots, S_{m}\right\}$ and $n$ time periods $T=\left\{T_{1}, T_{2}, \ldots, T_{n}\right\}$. Let $V=\left(v_{i j}\right)_{m \times n}$ be the comprehensive evaluation decision matrix, where $v_{i j}=\left\langle\alpha_{j} O_{i j}, \beta_{j} U_{i j}, \gamma_{j} R_{i j}\right\rangle$ is an evaluation value denoted by CINS. The following paragraphs propose the MCDM procedure that considers the integrated weights in order to rank and select the most trustworthy cloud service.

Step 1. Calculating the entropy value of the set $V=\left\{v_{i j}\right\}$. Using Eq. (19), the entropy value matrix of $V$ can be calculated as follows:

$$
\begin{aligned}
E(V)= & {\left[\begin{array}{cccc}
E\left(v_{11}\right) & E\left(v_{12}\right) & \cdots & E\left(v_{1 n}\right) \\
E\left(v_{21}\right) & E\left(v_{22}\right) & \cdots & E\left(v_{2 n}\right) \\
\vdots & \vdots & \ddots & \vdots \\
E\left(v_{m 1}\right) & E\left(v_{m 2}\right) & \cdots & E\left(v_{m n}\right)
\end{array}\right] } \\
= & {\left[\begin{array}{cccc}
1-d\left(v_{11}, A^{+}\right) & 1-d\left(v_{12}, A^{+}\right) & \cdots & 1-d\left(v_{1 n}, A^{+}\right) \\
1-d\left(v_{21}, A^{+}\right) & 1-d\left(v_{22}, A^{+}\right) & \cdots & 1-d\left(v_{2 n}, A^{+}\right) \\
\vdots & \vdots & \ddots & \vdots \\
1-d\left(v_{m 1}, A^{+}\right) & 1-d\left(v_{m 2}, A^{+}\right) & \cdots & 1-d\left(v_{m n}, A^{+}\right)
\end{array}\right] . }
\end{aligned}
$$

Step 2. Calculating the objective weight matrix of the time periods. The entropy of the time periods must first be calculated as follows:

$$
\begin{aligned}
E(T)= & {\left[\begin{array}{c}
E\left(T_{1}\right) \\
E\left(T_{2}\right) \\
\vdots \\
E\left(T_{n}\right)
\end{array}\right] } \\
= & {\left[\begin{array}{c}
1-\frac{2}{m(m-1)} \sum_{i=1}^{m} \sum_{k=i+1}^{m}\left|E\left(v_{i 1}\right)-E\left(v_{k 1}\right)\right| \\
1-\frac{2}{m(m-1)} \sum_{i=1}^{m} \sum_{k=i+1}^{m}\left|E\left(v_{i 2}\right)-E\left(v_{k 2}\right)\right| \\
1-\frac{2}{m(m-1)} \sum_{i=1}^{m} \sum_{k=i+1}^{m}\left|E\left(v_{i n}\right)-E\left(v_{k n}\right)\right|
\end{array}\right] . }
\end{aligned}
$$

Eq. (22) can easily calculate the objective weight matrix of the time periods:

$$
W(T)=\left[\begin{array}{c}
W\left(T_{1}\right) \\
W\left(T_{2}\right) \\
\vdots \\
W\left(T_{n}\right)
\end{array}\right]=\left[\begin{array}{c}
\left(1-E\left(T_{1}\right)\right) /\left(n-\sum_{i=1}^{n} E\left(T_{i}\right)\right) \\
\left(1-E\left(T_{2}\right)\right) /\left(n-\sum_{i=1}^{n} E\left(T_{i}\right)\right) \\
\vdots \\
\left(1-E\left(T_{n}\right)\right) /\left(n-\sum_{i=1}^{n} E\left(T_{i}\right)\right)
\end{array}\right] .
$$

Step 3. Using CINSWA operator to obtain CINS $y_{i}$ for service $S_{i}$ as follows:

$y_{i}=\operatorname{CINSWA}_{w}\left(v_{i 1}, v_{i 2}, \ldots, v_{i n}\right)$.

Step 4. Calculating the sort value of every candidate service. Inspired by the TOPSIS method [30], define a positive ideal CINS $A^{+}$and a negative ideal CINS $A^{-}$as follows:

$$
\begin{aligned}
A^{+} & =\left\langle O_{A^{+}}, U_{A^{+}}, R_{A^{+}}\right\rangle \\
& =\left\langle\max _{i=1}^{n}\left\{\alpha_{y_{i}} O_{y_{i}}\right\}, \min _{i=1}^{n}\left\{\beta_{y_{i}} U_{y_{i}}\right\}, \min _{i=1}^{n}\left\{\gamma_{y_{i}} R_{y_{i}}\right\}\right\rangle ; \\
A^{-} & =\left\langle O_{A^{-}}, U_{A^{-}}, R_{A^{-}}\right\rangle \\
& =\left\langle\min _{i=1}^{n}\left\{\alpha_{y_{i}} O_{y_{i}}\right\}, \max _{i=1}^{n}\left\{\beta_{y_{i}} U_{y_{i}}\right\}, \max _{i=1}^{n}\left\{\gamma_{y_{i}} R_{y_{i}}\right\}\right\rangle .
\end{aligned}
$$

Then, the Hamming distances between service $S_{i}$ and ideal CINSs $A^{+}$and $A^{-}$can be obtained using Eqs. (24) and (25):

$$
\begin{aligned}
d^{+}\left(y_{i}, A^{+}\right) & \\
= & 1-\left[\frac{1}{6}\left(\left|\alpha_{y_{i}} \inf O_{y_{i}}-\inf O_{A^{+}}\right|+\left|\alpha_{y_{i}} \sup O_{y_{i}}-\sup O_{A^{+}}\right|\right)\right. \\
& +\left|\beta_{y_{i}} \inf U_{y_{i}}-\inf U_{A^{+}}\right|+\left|\beta_{y_{i}} \sup U_{y_{i}}-\sup U_{A^{+}}\right| \\
& \left.+\left|\gamma_{y_{i}} \inf R_{y_{i}}-\inf R_{A^{+}}\right|+\left|\gamma_{y_{i}} \sup R_{y_{i}}-\sup R_{A^{+}}\right|\right],
\end{aligned}
$$

$d^{-}\left(y_{i}, A^{-}\right)$

$$
\begin{aligned}
= & 1-\left[\frac{1}{6}\left(\left|\alpha_{y_{i}} \inf O_{y_{i}}-\inf O_{A^{-}}\right|+\left|\alpha_{y_{i}} \sup O_{y_{i}}-\sup O_{A^{-}}\right|\right)\right. \\
& +\left|\beta_{y_{i}} \inf U_{y_{i}}-\inf U_{A^{-}}\right|+\left|\beta_{y_{i}} \sup U_{y_{i}}-\sup U_{A^{-}}\right| \\
& \left.+\left|\gamma_{y_{i}} \inf R_{y_{i}}-\inf R_{A^{-}}\right|+\left|\gamma_{y_{i}} \sup R_{y_{i}}-\sup R_{A^{-}}\right|\right] .
\end{aligned}
$$

Calculate the sort value of every candidate service according to Eq. (26):

$f_{i}^{*}=d^{-}\left(y_{i}, A^{-}\right) /\left(d^{+}\left(y_{i}, A^{+}\right)+d^{-}\left(y_{i}, A^{-}\right)\right)$. 

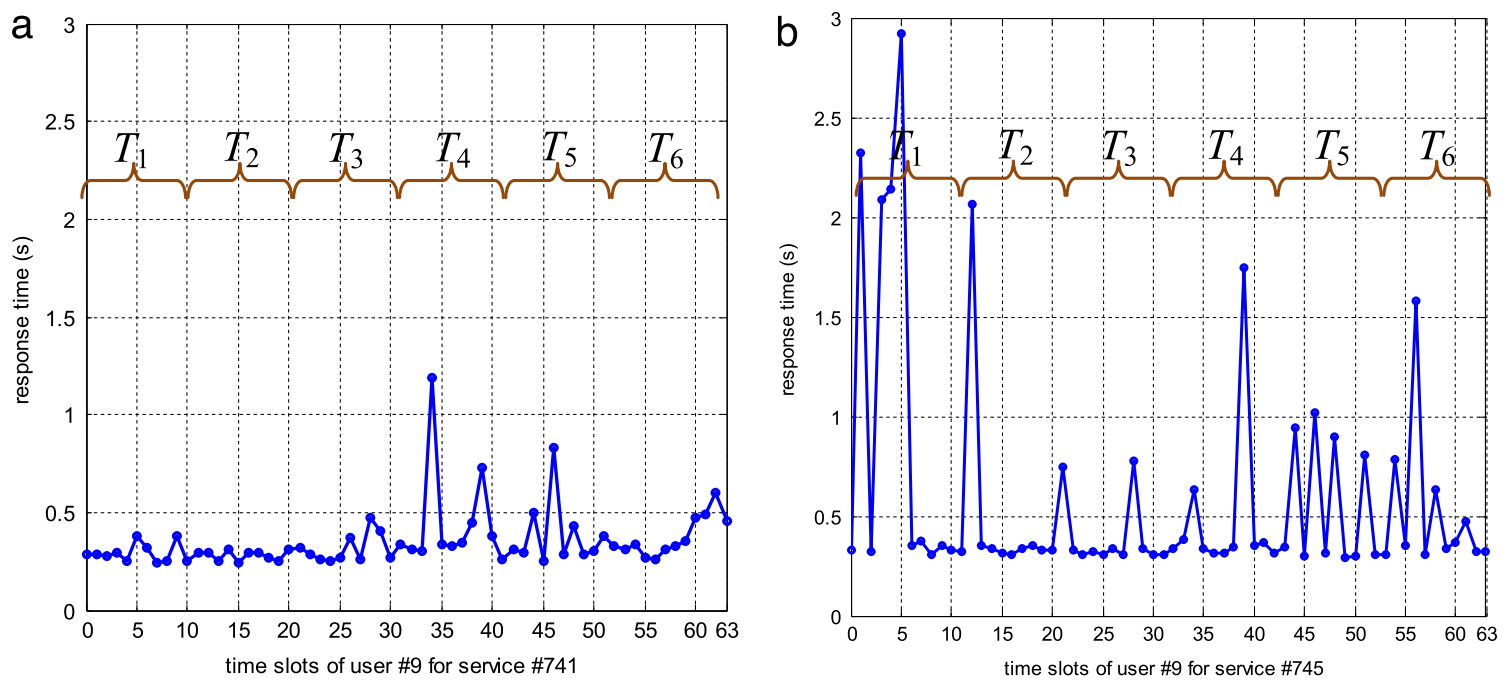

Fig. 2. Time period feature of response time. (a) service \#741; (b) service \#745.

Step 5. Obtaining the priority of service $S_{i}$ in accordance with $f_{i}^{*}$ and rank the candidate services. Then, the ranked list of services $S_{r 1} \succ S_{r 2} \succ \cdots \succ S_{r m-1} \succ S_{r m}$ will be recommended to the current user. The highest-ranked service in this list will have the optimal performance-costs and potential risks in $n$ time periods, and it will have a higher probability than lower-ranked services of being adopted by the current user.

\section{Experiments}

In order to demonstrate our approach in experiments, we used WS-DREAM dataset \#3 [11,86], which collected real-world QoS evaluations from PlanetLab [50], including response time and throughput, from 142 users on 4,532 services in 64 different timeslots. WS-DREAM datasets have been applied in much research concerned with cloud computing $[88,16,87]$. The analysis of response times, shown in Fig. 2, illustrates the fact that the services perform differently in six time periods. According to Fig. 2, user \#9 experienced a different QoS for service \#741 with service \#745. Service \#741 provided good performance in six time periods, whereas the performance of service \#745 was unsatisfactory in time periods $T_{1}$ and $T_{2}$.

Moreover, the coefficients of variation for response times of 3,873 services are larger than 1.0 , as shown in Fig. 3. The main reasons behind this finding are significant differences of client features among users, unpredictable network congestion and unexpected exceptions.

In the following experiments, let us consider a list of $m=8$ candidate services $S_{1}, S_{2}, \ldots, S_{8}$ with time periods $n=6$. Taking services \#741 through \#748 as an example, we employ the original evaluation data for response time in three numerical examples to demonstrate the proposed approach. The original data used in the experiments is provided online [24].

In order to measure the accuracy of our approach, the real response time experienced by current user is employed as an appropriate baseline for comparative analysis. The baseline sort

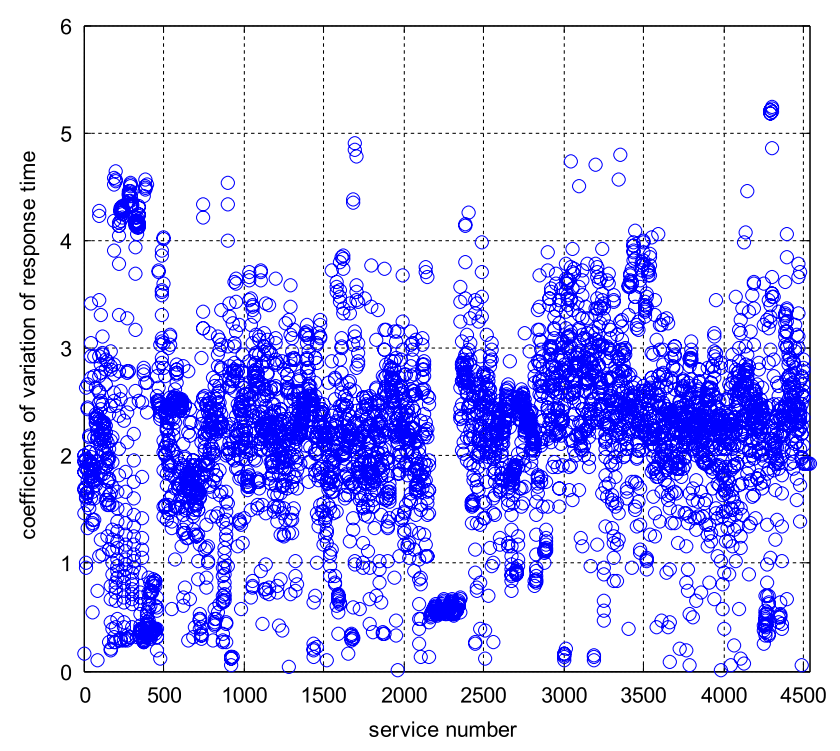

Fig. 3. Distribution of coefficients of variation for response times.

value of service $S_{i}(i=1,2, \ldots, m)$, noted as $f_{i}^{b}$, can be obtained by Eq. (27):

$f_{i}^{b}=\frac{\mid \text { risk }_{i}^{b}-\text { risk }^{b-} \mid}{\left|\operatorname{perf}_{i}^{b}-\operatorname{perf}^{b+}\right|+\mid \text { risk }_{i}^{b}-\text { risk }^{b-} \mid}$,

where $f_{i}^{b}$ is the baseline sort value of $S_{i}$; $\operatorname{perf}_{i}^{b}=\sum_{j=1}^{n}\left(\alpha_{i} \times\right.$ $\left.\sum_{k=(j-1) \times d+1}^{j \times d} \operatorname{perf}_{k}\right) / \sum_{j=1}^{n} \alpha_{i}$ and risk $k_{i}^{b}=\sum_{j=1}^{n}\left(\gamma_{i} \times \sum_{k=(j-1) \times d+1}^{j \times d}\right.$ $\left.r i s k_{k}\right) / \sum_{j=1}^{n} \gamma_{i}$ represent the total response time and the total risk value of $S_{i}$ aggregated with weights in all of time periods, respectively; $\alpha_{i}$ and $\gamma_{i}$ are the tradeoff coefficients for performance-cost and risks respectively in $T_{i}$; $\operatorname{perf}_{k}$ and risk $_{k}$ represent the actual response time and risk value experienced by the current user in the $k$ th timeslot; risk $k^{b-}=\max _{i=1}^{n}\left\{\right.$ risk $\left._{i}^{b}\right\}$, perf $^{b+}=\max _{i=1}^{n}\left\{\right.$ perf $\left._{i}^{b}\right\}$. The order of $S_{i}$ in baseline ranking can be obtained in accordance with

Table 2

Parameters of time periods in WS-DREAM.

\begin{tabular}{|c|c|c|c|c|c|c|}
\hline Time periods & $T_{1}$ & $T_{2}$ & $T_{3}$ & $T_{4}$ & $T_{5}$ & $T_{6}$ \\
\hline Range of timeslots & {$[0,10]$} & {$[11,20]$} & {$[21,30]$} & {$[31,40]$} & {$[41,51]$} & {$[52,63]$} \\
\hline Line number ranges & {$[1,1139]$} & {$[1140,2146]$} & {$[2147,3135]$} & {$[3136,4105]$} & {$[4106,5159]$} & {$[5160,6294]$} \\
\hline
\end{tabular}




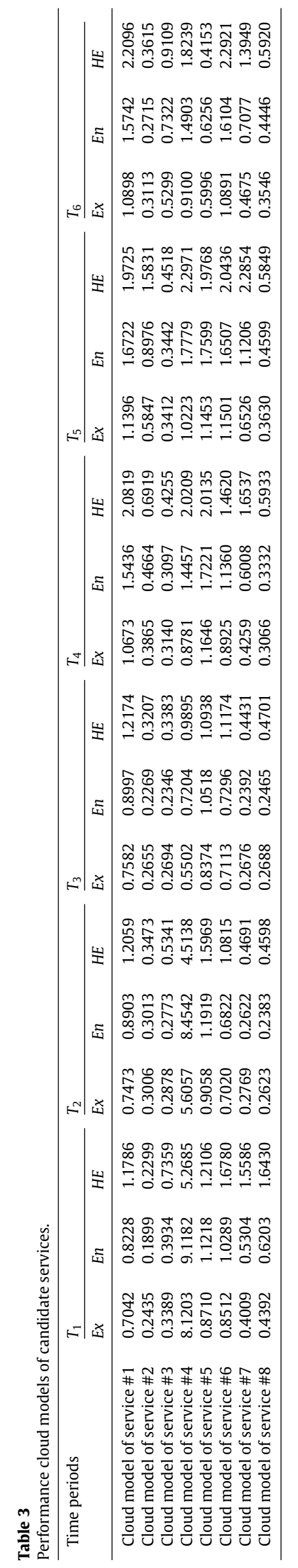




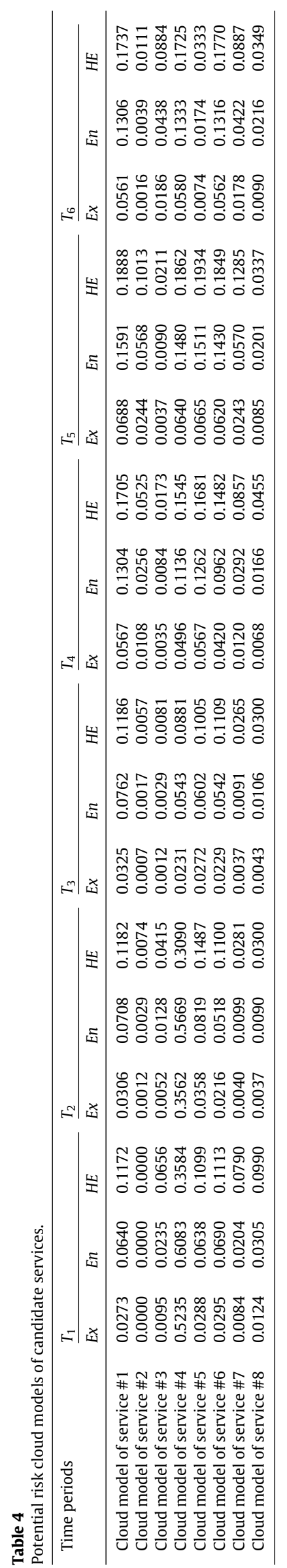




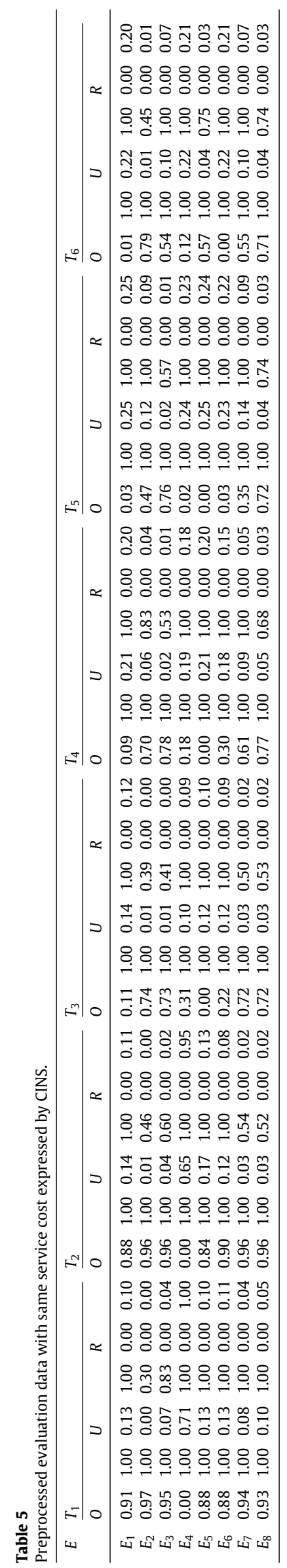




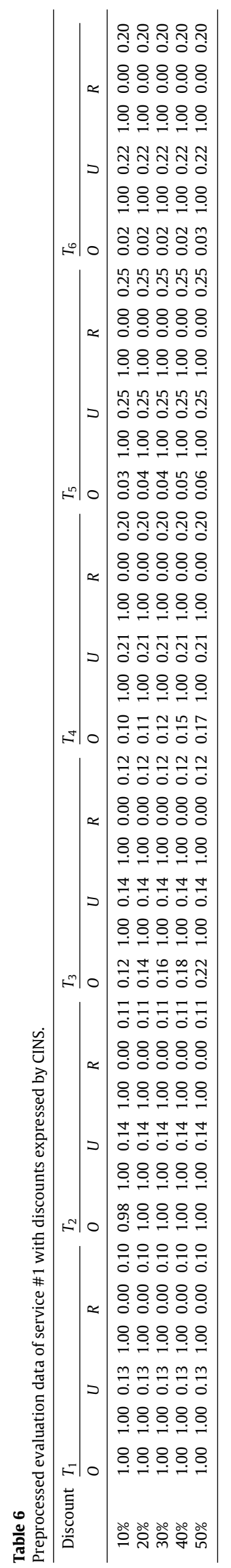


$f_{i}^{b}$. The difference degree, noted as $D$, is defined to compare our ranked service list and the baseline list as follows:

$D=\sum_{i=1}^{m}\left|d_{i}\right|=\sum_{i=1}^{m} \frac{\left|R_{i}-B_{i}\right|}{B_{i}}$,

where $R_{i}$ represents the ranking order of $S_{i}$ obtained by our approach; $B_{i}$ represents the order of $S_{i}$ in baseline ranking; $d_{i}$ represents the relative difference of $S_{i}$. Obviously, smaller $D$ means better accuracy.

\subsection{Preprocessing evaluation data}

The original data is divided into six time periods. Table 2 shows the range of timeslots and the line number ranges in datasets for every time period.

Targeting response time, we evaluate the potential risks of services with Eq. (29):

$v_{i}^{t}= \begin{cases}0, & r t_{i}<\zeta \\ \left(\delta \times\left(r t_{i}-\zeta\right)\right) / \zeta, & \zeta \leq r t_{i} \leq(\zeta(1+\delta)) / \delta \\ 1, & (\zeta(1+\delta)) / \delta<r t_{i},\end{cases}$

where $\zeta$ is the user's expectation of response time and $r t_{i}$ represents the response time experienced by user $i$. If $r t_{i} \leq \zeta$, the $i$ th user considers this service to be risk-free. $\delta$ is an adjustment factor that determines the tolerable range for response time. According to the "2-5-10" principle [14] of response time in software testing analysis, we can set $\zeta=2$ s and $\delta=0.25$. In addition, $\gamma$ is set as 0.1 .

In experiments, user \#9 is viewed as the current user. Assume service \#740 is in $S^{\circ}$ and the nearest neighbors of user \#9 are identified with $\operatorname{sim}^{\text {th }}=0.2$. Then, on the basis of evaluation data from nearest neighbors, the cloud models of performance and potential risks of candidate services can be obtained according to Eqs. (11)-(13), shown in Tables 3 and 4. The original evaluation data can be transformed into CINS, as shown in Table 5 .

Because no cost data exists for services in the dataset, assume that the costs of all services are identical. Considering that in the MCDM procedure, the cost of services plays a part with the performance of services together, namely performance-cost. Table 3 demonstrates that there is enough diversity in the performance data of real services, which can ensure the effectiveness of our outcome. In addition, we assume that service \#1 attempts to improve its performance-cost ratio by offering price discounts. Table 6 displays the preprocessed evaluation data for service \#1 with different discounts expressed by CINS.

\subsection{Experiment in risk-sensitive service selection mode}

Example 1. Assume that a large-scale security company is ready to purchase a cloud service to store massive amounts of stock trading data; the service should have fairly high trustworthiness with a high performance-cost ratio and low potential risks. Considering that the peak stock trading time is from 9:30 to $11: 30$ and from 13:00 to 15:00 every working day, the trustworthiness evaluation of the cloud service is more important during these two time periods than in other time periods. Based on this analysis of the user's requirements, we can define tradeoff coefficients for the time periods, as shown in Table 7 .

According to the approach proposed in Section 6, the entropy value of the set $V=\left\{v_{i j}\right\}$ can be obtained using data from Table 5, yielding the results shown in Table 8.

Table 9 presents the entropies and weights of the time periods.

Table 10 shows the aggregation values of candidate services in the six target time periods.
Table 7

Tradeoff coefficients for time periods in Example 1.

\begin{tabular}{lllllll}
\hline$F$ & $F_{1}$ & $F_{2}$ & $F_{3}$ & $F_{4}$ & $F_{5}$ & $F_{6}$ \\
\hline$\alpha$ & 0 & 0.6 & 0.6 & 0.6 & 0.6 & 0 \\
$\beta$ & 0 & 0.8 & 1 & 1 & 0.8 & 0 \\
$\gamma$ & 0 & 0.8 & 1 & 1 & 0.8 & 0 \\
\hline
\end{tabular}

Table 8

Entropy values for set $V$ in Example 1.

\begin{tabular}{lllllll}
\hline$E(V)$ & $T_{1}$ & $T_{2}$ & $T_{3}$ & $T_{4}$ & $T_{5}$ & $T_{6}$ \\
\hline$E\left(V_{1}\right)$ & 0.5918 & 0.8295 & 0.7747 & 0.7690 & 0.7606 & 0.5918 \\
$E\left(V_{2}\right)$ & 0.5918 & 0.9230 & 0.9213 & 0.8518 & 0.8099 & 0.5918 \\
$E\left(V_{3}\right)$ & 0.5918 & 0.8991 & 0.9180 & 0.9051 & 0.8974 & 0.5918 \\
$E\left(V_{4}\right)$ & 0.5918 & 0.6960 & 0.7967 & 0.7803 & 0.7606 & 0.5918 \\
$E\left(V_{5}\right)$ & 0.5918 & 0.8276 & 0.7629 & 0.7594 & 0.7575 & 0.5918 \\
$E\left(V_{6}\right)$ & 0.5918 & 0.8306 & 0.7874 & 0.7925 & 0.7618 & 0.5918 \\
$E\left(V_{7}\right)$ & 0.5918 & 0.9100 & 0.9042 & 0.8205 & 0.7997 & 0.5918 \\
$E\left(V_{8}\right)$ & 0.5918 & 0.9133 & 0.8997 & 0.8801 & 0.8670 & 0.5918 \\
\hline
\end{tabular}

Table 9

Entropies and the weights of time periods in Example 1.

\begin{tabular}{lllllll}
\hline Time periods & $T_{1}$ & $T_{2}$ & $T_{3}$ & $T_{4}$ & $T_{5}$ & $T_{6}$ \\
\hline$E\left(T_{i}\right)$ & 1.0000 & 0.8503 & 0.8800 & 0.9247 & 0.9427 & 1.0000 \\
$W\left(T_{i}\right)$ & 0.0000 & 0.3721 & 0.2983 & 0.1873 & 0.1423 & 0.0000 \\
\hline
\end{tabular}

Table 10

Aggregation values of candidate services in Example 1.

\begin{tabular}{ll}
\hline$Y$ & Aggregation values \\
\hline$y_{1}$ & $\langle[0.2678,1.0000],[0.1460,0.8916],[0.0000,0.1283]\rangle$ \\
$y_{2}$ & $\langle[0.4733,1.0000],[0.0138,0.4884],[0.0000,0.0085]\rangle$ \\
$y_{3}$ & $\langle[0.5017,1.0000],[0.0187,0.4634],[0.0000,0.0109]\rangle$ \\
$y_{4}$ & $\langle[0.0825,1.0000],[0.2304,0.8916],[0.0000,0.2479]\rangle$ \\
$y_{5}$ & $\langle[0.2312,1.0000],[0.1486,0.8916],[0.0000,0.1265]\rangle$ \\
$y_{6}$ & $\langle[0.3091,1.0000],[0.1281,0.8916],[0.0000,0.0979]\rangle$ \\
$y_{7}$ & $\langle[0.4562,1.0000],[0.0401,0.5767],[0.0000,0.0229]\rangle$ \\
$y_{8}$ & $\langle[0.4962,1.0000],[0.0314,0.5154],[0.0000,0.0179]\rangle$ \\
\hline
\end{tabular}

The sort values of candidate services can be calculated using Eqs. (24)-(26), with the results shown in Table 11.

By comparing the sort values, the ranked list of services is finally obtained: $S_{3} \succ S_{2} \succ S_{8} \succ S_{7} \succ S_{6} \succ S_{1} \succ S_{5} \succ S_{4}$. In other words, service \#3 is the optimal candidate and will be recommended to the large-scale security company. The difference degree between our approach and the baseline is calculated according to Eqs. (28) and (27)shown in Table 12.

The result demonstrates that the difference degree is much less than 1.0. What is more important, the top four services in our services list are identical to the baseline list. In addition, the difference degree can be further decreased if there is enough training data used in searching the nearest neighbors of current user.

\subsection{Experiment in performance-cost-sensitive service selection mode}

Example 2. Assume that a small- and medium-sized logistics company is preparing to purchase a cloud service host to deploy their express delivery query application. The budget is very limited, and no highly confidential data is involved; therefore, the company desires the performance-cost ratio of the cloud host service to be as high as possible, on the premise that the potential risks are sufficiently low. The anticipated peak visiting time for this application is from 9:00 to 17:00 every working day. Based on this analysis of the user's requirements, we can define tradeoff coefficients for time periods as shown in Table 13.

The entropy value $V$ can be obtained according to Table 5 and is shown in Table 14. 
Table 11

Sort values of candidate services in Example 1.

\begin{tabular}{|c|c|c|c|c|c|c|c|c|}
\hline Candidate services & $S_{1}$ & $S_{2}$ & $S_{3}$ & $S_{4}$ & $S_{5}$ & $S_{6}$ & $S_{7}$ & $S_{8}$ \\
\hline$d^{-}\left(y_{i}, A^{-}\right)$ & 0.1515 & 0.0097 & 0.0004 & 0.2164 & 0.1578 & 0.1366 & 0.0324 & 0.0133 \\
\hline$d^{+}\left(y_{i}, A^{+}\right)$ & 0.0450 & 0.1884 & 0.1961 & 0.0199 & 0.0387 & 0.0599 & 0.1641 & 0.1832 \\
\hline Sort value & 0.2288 & 0.9509 & 0.9980 & 0.0843 & 0.1971 & 0.3048 & 0.8351 & 0.9324 \\
\hline Ranking order & 6 & 2 & 1 & 8 & 7 & 5 & 4 & 3 \\
\hline
\end{tabular}

Table 12

Comparative analysis based on baseline in Example 1.

\begin{tabular}{|c|c|c|c|c|c|c|c|c|}
\hline Candidate services & $S_{1}$ & $S_{2}$ & $S_{3}$ & $S_{4}$ & $S_{5}$ & $S_{6}$ & $S_{7}$ & $S_{8}$ \\
\hline Baseline sort value & 0.2159 & 0.9577 & 1.0000 & 0.0000 & 0.1180 & 0.0507 & 0.4391 & 0.5005 \\
\hline Baseline ranking order & 5 & 2 & 1 & 8 & 6 & 7 & 4 & 3 \\
\hline$d_{i}$ & 0.2000 & 0.0000 & 0.0000 & 0.0000 & 0.1667 & 0.2857 & 0.0000 & 0.0000 \\
\hline$D$ & 0.6524 & & & & & & & \\
\hline
\end{tabular}

Table 13

Tradeoff coefficients for time periods in Example 2.

\begin{tabular}{lllllll}
\hline$F$ & $F_{1}$ & $F_{2}$ & $F_{3}$ & $F_{4}$ & $F_{5}$ & $F_{6}$ \\
\hline$\alpha$ & 0.8 & 0.8 & 1 & 1 & 1 & 0.8 \\
$\beta$ & 0.6 & 0.6 & 0.6 & 0.6 & 0.6 & 0.6 \\
$\gamma$ & 0.6 & 0.6 & 0.6 & 0.6 & 0.6 & 0.6 \\
\hline
\end{tabular}

Table 14

Entropy values of set $V$ in Example 2.

\begin{tabular}{lllllll}
\hline$E(V)$ & $T_{1}$ & $T_{2}$ & $T_{3}$ & $T_{4}$ & $T_{5}$ & $T_{6}$ \\
\hline$E\left(V_{1}\right)$ & 0.8303 & 0.8295 & 0.7747 & 0.769 & 0.7606 & 0.7608 \\
$E\left(V_{2}\right)$ & 0.9501 & 0.923 & 0.9213 & 0.8518 & 0.8099 & 0.9168 \\
$E\left(V_{3}\right)$ & 0.8601 & 0.8991 & 0.918 & 0.9051 & 0.8974 & 0.8153 \\
$E\left(V_{4}\right)$ & 0.6883 & 0.696 & 0.7967 & 0.7803 & 0.7606 & 0.7722 \\
$E\left(V_{5}\right)$ & 0.8299 & 0.8276 & 0.7629 & 0.7594 & 0.7575 & 0.8554 \\
$E\left(V_{6}\right)$ & 0.8298 & 0.8306 & 0.7874 & 0.7925 & 0.7618 & 0.759 \\
$E\left(V_{7}\right)$ & 0.8324 & 0.91 & 0.9042 & 0.8205 & 0.7997 & 0.8162 \\
$E\left(V_{8}\right)$ & 0.8318 & 0.9133 & 0.8997 & 0.8801 & 0.867 & 0.867
\end{tabular}

Table 15

Entropies and weights of time periods in Example 2.

\begin{tabular}{lllllll}
\hline Time periods & $T_{1}$ & $T_{2}$ & $T_{3}$ & $T_{4}$ & $T_{5}$ & $T_{6}$ \\
\hline$E\left(T_{i}\right)$ & 0.8576 & 0.8503 & 0.8800 & 0.9247 & 0.9427 & 0.9532 \\
$W\left(T_{i}\right)$ & 0.2407 & 0.2531 & 0.2029 & 0.1274 & 0.0968 & 0.0792 \\
\hline
\end{tabular}

Table 16

Aggregation values of candidate services in Example 2.

\begin{tabular}{ll}
\hline$Y$ & Aggregation values \\
\hline$y_{1}$ & $\langle[0.4821,1.0000],[0.0957,0.6000],[0.0000,0.0819]\rangle$ \\
$y_{2}$ & $\langle[0.7264,1.0000],[0.0000,0.2797],[0.0000,0.0000]\rangle$ \\
$y_{3}$ & $\langle[0.7436,1.0000],[0.0190,0.3681],[0.0000,0.0112]\rangle$ \\
$y_{4}$ & $\langle[0.1063,1.0000],[0.1948,0.6000],[0.0000,0.2219]\rangle$ \\
$y_{5}$ & $\langle[0.4655,1.0000],[0.0833,0.5865],[0.0000,0.0694]\rangle$ \\
$y_{6}$ & $\langle[0.5109,1.0000],[0.0872,0.6000],[0.0000,0.0692]\rangle$ \\
$y_{7}$ & $\langle[0.6912,1.0000],[0.0331,0.4461],[0.0000,0.0181]\rangle$ \\
$y_{8}$ & $\langle[0.7368,1.0000],[0.0278,0.4036],[0.0000,0.0159]\rangle$ \\
\hline
\end{tabular}

Table 15 presents the entropies and weights of the time periods.

Table 16 shows the aggregation values of the candidate services in the six time periods.

Table 17 shows the sort values of the candidate services.

The ranked list of services is obtained by comparing the sort values: $S_{2} \succ S_{3} \succ S_{8} \succ S_{7} \succ S_{6} \succ S_{1} \succ S_{5} \succ S_{4}$. In other words, service \#2 is the optimal candidate and will be recommended to the logistics company. The difference degree between our approach and the baseline is shown in Table 18. According to Table 18, the sort order of six services in our ranked list is identical to the baseline list, including the top four services.

\subsection{Experiment in low price competition mode}

Example 3. Assume that service \#1 adopts a low price strategy to improve its performance-cost ratio. When service \#1 offers different discounts from $10 \%$ to $50 \%$, its performance-cost ratio will change as shown in Table 6 . In this case, we can again utilize our approach to help the security company in Example 1 and the logistics company in Example 2 to make their decisions. Table 19 displays the sort values of the candidate services in low price competition mode for comparative analysis between our approach and the baseline.

Table 19 shows that service \#1 fails to increase its probability to be an optimal service or to obtain a distinct advantage, although it achieves an attractive performance-cost ratio. The service selection approach based on CINS consistently maintains the absolute dominance of service \#3 for security company and the advantage of service \#2 for logistics company.

\subsection{Analysis and discussion}

These experiments illustrate the following merits of the proposed approach:

(1) Support for user preferences regarding time periods improves consumer satisfaction. Table 5 indicates that the evaluation data of service \#3 in Example 1 is far from outstanding in time periods $T_{1}$ and $T_{6}$ in comparison with the other services, but service \#3 becomes the optimal candidate by relying on its advantages in time periods $T_{3}$ and $T_{4}$. The security company does not care at all about time periods $T_{1}$ and $T_{6}$, while time periods $T_{3}$ and $T_{4}$ cover the period of stock exchange. Moreover, the tradeoff coefficients are set as $\alpha<\beta$ and $\alpha<\gamma$ in time periods $T_{2} \sim T_{5}$ because the security company is more sensitive to potential risks than to performance-costs. An accurate analysis of time periods can help the security company find the most trustworthy candidate service.

(2) Different service selection modes can produce ideal recommendation results. In Example 1's risk-sensitive service selection mode, the assessment of potential risks plays a more important role than performance-cost ratio in evaluating candidate services, which leads to service \#3 being judged as the most trustworthy candidate service. However, Example 2's performance-cost-sensitive service selection mode shows an entirely different recommendation result, in which the service \#2 is the most trustworthy candidate.

(3) Prevent low-quality services with high performance-cost ratios from achieving an absolute advantage in competition with other services. In practice, some service providers adopt this low-price strategy to improve their performance-cost 
Table 17

Sort values of candidate services in Example 2.

\begin{tabular}{|c|c|c|c|c|c|c|c|c|}
\hline Candidate services & $S_{1}$ & $S_{2}$ & $S_{3}$ & $S_{4}$ & $S_{5}$ & $S_{6}$ & $S_{7}$ & $S_{8}$ \\
\hline$d^{-}\left(y_{i}, A^{-}\right)$ & 0.1266 & 0.0029 & 0.0198 & 0.2290 & 0.1229 & 0.1182 & 0.0450 & 0.0291 \\
\hline$d^{+}\left(y_{i}, A^{-}\right)$ & 0.0791 & 0.2029 & 0.1860 & 0.0233 & 0.0828 & 0.0875 & 0.1607 & 0.1767 \\
\hline Sort value & 0.3847 & 0.9861 & 0.9039 & 0.0924 & 0.4024 & 0.4252 & 0.7812 & 0.8588 \\
\hline Ranking order & 7 & 1 & 2 & 8 & 6 & 5 & 4 & 3 \\
\hline
\end{tabular}

Table 18

Comparative analysis based on baseline in Example 2.

\begin{tabular}{|c|c|c|c|c|c|c|c|c|}
\hline Candidate services & $S_{1}$ & $S_{2}$ & $S_{3}$ & $S_{4}$ & $S_{5}$ & $S_{6}$ & $S_{7}$ & $S_{8}$ \\
\hline Baseline sort value & 0.4931 & 1.0000 & 0.9265 & 0.0000 & 0.2861 & 0.2646 & 0.7250 & 0.7549 \\
\hline Baseline ranking order & 5 & 1 & 2 & 8 & 6 & 7 & 4 & 3 \\
\hline$d_{i}$ & 0.4000 & 0.0000 & 0.0000 & 0.0000 & 0.0000 & 0.2857 & 0.0000 & 0.0000 \\
\hline$D$ & 0.6857 & & & & & & & \\
\hline
\end{tabular}

Table 19

Comparative analysis in low price competition mode.

\begin{tabular}{|c|c|c|c|c|c|c|c|c|c|c|c|}
\hline \multirow[t]{2}{*}{ Discounts } & \multirow{2}{*}{$\begin{array}{l}\text { Application } \\
\text { scenarios }\end{array}$} & \multicolumn{8}{|c|}{ Candidate services } & \multirow{2}{*}{$\begin{array}{l}\text { Ranking order } \\
\text { of } S_{1}\end{array}$} & \multirow{2}{*}{$\begin{array}{l}\text { Ranking order } \\
\text { in baseline }\end{array}$} \\
\hline & & $S_{1}$ & $S_{2}$ & $S_{3}$ & $S_{4}$ & $S_{5}$ & $S_{6}$ & $S_{7}$ & $S_{8}$ & & \\
\hline \multirow{5}{*}{$\begin{array}{l}\text { Security } \\
\text { company }\end{array}$} & $10 \%$ & 0.2610 & 0.9509 & 0.9980 & 0.0843 & 0.1972 & 0.3048 & 0.8351 & 0.9324 & 6 & 5 \\
\hline & $20 \%$ & 0.2716 & 0.9510 & 0.9980 & 0.0844 & 0.1975 & 0.3051 & 0.8352 & 0.9325 & 6 & 5 \\
\hline & $30 \%$ & 0.2756 & 0.9510 & 0.9980 & 0.0846 & 0.1979 & 0.3055 & 0.8353 & 0.9326 & 6 & 5 \\
\hline & $40 \%$ & 0.2809 & 0.9510 & 0.9980 & 0.0848 & 0.1984 & 0.3059 & 0.8355 & 0.9327 & 6 & 5 \\
\hline & $50 \%$ & 0.2883 & 0.9511 & 0.9980 & 0.0850 & 0.1990 & 0.3065 & 0.8357 & 0.9329 & 6 & 5 \\
\hline \multirow{5}{*}{$\begin{array}{l}\text { Logistics } \\
\text { company }\end{array}$} & $10 \%$ & 0.4461 & 0.9861 & 0.9039 & 0.0925 & 0.4025 & 0.4253 & 0.7812 & 0.8587 & 5 & 5 \\
\hline & $20 \%$ & 0.4564 & 0.9861 & 0.9038 & 0.0925 & 0.4028 & 0.4255 & 0.7812 & 0.8587 & 5 & 5 \\
\hline & $30 \%$ & 0.4594 & 0.9861 & 0.9037 & 0.0926 & 0.4032 & 0.4258 & 0.7812 & 0.8587 & 5 & 5 \\
\hline & $40 \%$ & 0.4634 & 0.9862 & 0.9036 & 0.0928 & 0.4037 & 0.4261 & 0.7812 & 0.8586 & 5 & 5 \\
\hline & $50 \%$ & 0.4690 & 0.9862 & 0.9034 & 0.0930 & 0.4043 & 0.4266 & 0.7812 & 0.8586 & 5 & 5 \\
\hline
\end{tabular}

ratio for low-quality services. In our approach, the low-price strategy can increase the popularity of low-quality services to some extent, but it does not help these services to dominate their competition based solely on a malignant price war, as shown in Table 19. The poor experience quality of a cloud service in indicators such as efficiency, availability or information security necessarily gives it high marks in the assessment of potential risks, which greatly reduces its probability of being a most trustworthy candidate. For example, according to Table 19, cloud service \#1 has a fairly high performance-cost ratio; however, its assessment of potential risks shows very poor results. As a result, service \#1 does not earn ideal sort values in Example 3.

(4) The entropy weight measure method for CINS proposed in this paper can effectively manifest the differences between candidate services. The larger the entropy of a time period is, the smaller its weight should be. Although time period $T_{4}$ is very important to the security company in Example 1, the weights assigned to it by our method are much lower than the weight of time period $T_{2}$, as shown in Table 9. The evaluation data of the candidate services in time period $T_{4}$ are very similar, such that assigning more weight to it does not help to identify the differences between candidates and find the most trustworthy service.

\section{Conclusion}

In the uncertain cloud computing environment, fluctuating QoS, flexible service pricing and complicated potential risks have always presented challenges to trustworthy service selection. Massive amounts of evaluation data regarding QoS, service cost and potential risks have created a critical issue in facilitating users to select trustworthy cloud services with tradeoffs between performance-costs and potential risks from abundant candidates.
Experiments based on real-world datasets demonstrate that the time period feature of cloud services can provide a new perspective to help solve this problem by accurately assessing the performance, cost and potential risks for cloud services.

This paper proposes a time series analysis approach to select trustworthy cloud service combined with INS theory, in which the original evaluation data about the performance-costs and potential risks of cloud services are preprocessed via cloud model method and measured with INS. In order to calculate and compare candidate services while supporting tradeoffs between performance-costs and potential risks during different time periods, we propose the CINS theory. We design its calculation rules and operators, and provide theoretical proofs in support. To address the problem of selecting trustworthy services with tradeoffs between performance-costs and potential risks in multiple time periods, we formulate the question as a MCDM problem of creating a ranked services list, and we develop a CINS ranking method to solve the problem. Experiments consisting of three numerical examples based on an appropriate baseline for comparative analysis demonstrate that the proposed approach can work effectively in both the risk-sensitive service selection mode and the performance-cost-sensitive service selection mode, and it can also prevent malignant price competition launched by lowquality services. This paper can provide a strong decision support approach for time-aware trustworthy service selection problem in cloud environment.

\section{Acknowledgments}

The authors acknowledge the contribution of reviewers and thank them for their valuable comments and constructive suggestions to improve the manuscript. This work was partially supported by National Natural Science Foundation of China (Nos. 61572525 and 71501192). 


\section{Appendix A. Supplementary data}

Supplementary material related to this article can be found online at http://dx.doi.org/10.1016/j.jpdc.2016.05.008.

\section{References}

[1] J. Allen, S. Singh, Neurofuzzy and neutrosophic approach to compute the rate of change in new economies, in: Proc. of the First International Conference on Neutrosophy, Neutrosophic Logic, Neutrosophic Set, Neutrosophic Probability and Statistics, University of New Mexico, 2001, pp. 56-62.

[2] A. Ansari, R. Biswas, S. Aggarwal, Proposal for applicability of neutrosophic set theory in medical AI, Int. J. Comput. Appl. 27 (5) (2011) 5-11.

[3] K.T. Atanassov, Intuitionistic fuzzy sets, Fuzzy Sets and Systems 20 (1) (1986) 87-96.

[4] K. Atanassov, G. Gargov, Interval valued intuitionistic fuzzy sets, Fuzzy Sets and Systems 31 (3) (1989) 343-349.

[5] E. Ayday, F. Fekri, Iterative trust and reputation management using belief propagation, IEEE Trans. Dependable Secure Comput. 9 (3) (2012) 375-386

[6] N. Brender, I. Markov, Risk perception and risk management in cloud computing: Results from a case study of Swiss companies, Int. J. Inf. Manage. 33 (5) (2013) 726-733.

[7] P. Casas, R. Schatz, Quality of experience in cloud services: survey and measurements, Comput. Netw. 68 (2014) 149-165.

[8] P. Chen, An interval estimation for the number of signals, Signal Process. 85 (8) (2005) 1623-1633.

[9] J. Chen, Y. Liu, D. Li, Enhancing recommender diversity using gaussian cloud transformation, Int. J. Uncertain. Fuzziness Knowl.-Based Syst. 23 (04) (2015) 521-544.

[10] China Cloud Computing Promotion and Policy Forum, The evaluation results of trusted services authentication, October 2015. URL: http://www.3cpp.org/news/201.html.

[11] Chinese University of Hong Kong, WS-DREAM, February 2016. URL: http://www.wsdream.net/dataset.html.

[12] Cloud Security Alliance, Cloud Controls Matrix v3.0, October. 2015. URL: https://cloudsecurityalliance.org/download/cloud-controls-matrix-v3/.

[13] Cloud Security Alliance, CSA Security, Trust \& Assurance Registry (STAR), October 2015. URL: https://cloudsecurityalliance.org/star/.

[14] Databaseskill.com, LoadRunner did not tell you, October 2015. URL: http://www.Databaseskill.com/471323/.

[15] K. Dempsey, N.S. Chawla, A. Johnson, R. Johnston, A.C. Jones, A. Orebaugh, M. Scholl, K. Stine, Information Security Continuous Monitoring (ISCM) for Federal Information Systems and Organizations, National Institute of Standards and Technology Special Publication, 2012, 800-137.

[16] S. Ding, S.L. Yang, Y.T. Zhang, C.Y. Liang, C.Y. Xia, Combining QoS prediction and customer satisfaction estimation to solve cloud service trustworthiness evaluation problems, Knowl.-Based Syst. 56 (2014) 216-225.

[17] S. Drissi, H. Houmani, H. Medromi, Survey: Risk assessment for cloud computing, Int. J. Adv. Comput. Sci. Appl. 4 (12) (2013) 143-148.

[18] S.K. Garg, S. Versteeg, R. Buyya, A framework for ranking of cloud computing services, Future Gener. Comput. Syst. 29 (4) (2013) 1012-1023.

[19] N. Ghosh, S.K. Ghosh, S.K. Das, SelCSP: A framework to facilitate selection of cloud service providers, IEEE Trans. Cloud Comput. 3 (1) (2015) 66-79.

[20] M. Godse, S. Mulik, An approach for selecting software-as-a-service (SaaS) product, in: Proc. of IEEE International Conference on Cloud Computing, CLOUD'09, IEEE, 2009, pp. 155-158.

[21] Y. Guo, H.D. Cheng, New neutrosophic approach to image segmentation, Pattern Recognit. 42 (5) (2009) 587-595.

[22] M.L. Hale, R. Gamble, Secagreement: Advancing security risk calculations in cloud services, in: Proc. of 2012 IEEE Eighth World Congress on Services, SERVICES, IEEE, 2012, pp. 133-140.

[23] K. Hanbay, M.F. Talu, Segmentation of SAR images using improved artificial bee colony algorithm and neutrosophic set, Appl. Soft Comput. 21 (2014) 433-443.

[24] HIEU2011, Data for CINS, February 2016. URL: http://pan.baidu.com/s/ 1 toxg5V.

[25] Y. Hu, Q. Peng, X. Hu, A time-aware and data sparsity tolerant approach for web service recommendation, in: Proc. of 2014 IEEE International Conference on Web Services, ICWS, IEEE, 2014, pp. 33-40.

[26] Y. Huo, Y. Zhuang, S. Ni, Fuzzy trust evaluation based on consistency intensity for cloud services, Kybernetes 44 (1) (2015) 7-24.

[27] M. Jarschel, D. Schlosser, S. Scheuring, T. Hoßfeld, Gaming in the clouds: QoE and the users' perspective, Math. Comput. Modelling 57 (11) (2013) 2883-2894.

[28] B.P. Joshi, S. Kumar, Fuzzy time series model based on intuitionistic fuzzy sets for empirical research in stock market, Int. J. Appl. Evol. Comput. 3 (4) (2012)

$71-84$.
[29] N.N. Joshi, J.H. Lambert, Equity metrics with risk, performance, and cost objectives for the prioritization of transportation projects, IEEE Trans. Eng. Manage. 54 (3) (2007) 539-547.

[30] F.R.L. Junior, L. Osiro, L.C.R. Carpinetti, A comparison between fuzzy AHP and fuzzy TOPSIS methods to supplier selection, Appl. Soft Comput. 21 (2014) 194-209.

[31] B. Kavitha, S. Karthikeyan, P.S. Maybell, An ensemble design of intrusion detection system for handling uncertainty using neutrosophic logic classifier, Knowl.-Based Syst. 28 (2012) 88-96.
[32] K.M. Khan, Q. Malluhi, Establishing trust in cloud computing, IT Prof. 12 (5) (2010) 20-27.

[33] M. Khoshnevisan, S. Bhattacharya, A short note on financial data set detection using neutrosophic probability, in: Proc. of the First International Conference on Neutrosophy, Neutrosophic Logic, Neutrosophic Set, Neutrosophic Probability and Statistics, University of New Mexico, 2001, pp. 75-80.

[34] K. Li, Optimal load distribution for multiple heterogeneous blade servers in a cloud computing environment, J. Grid Comput. 11 (1) (2013) 27-46.

[35] D.Y. Li, H.J. Meng, X.M. Shi, Membership clouds and membership cloud generators, J. Comput. Res. Dev. 32 (6) (1995) 15-20.

[36] S. Lins, S. Thiebes, S. Schneider, A. Sunyaev, What is really going on at your cloud service provider? Creating trustworthy certifications by continuous auditing, in: Proc. of 2015 48th Hawaii International Conference on System Sciences, HICSS, IEEE, 2015, pp. 5352-5361.

[37] P. Liu, Y. Chu, Y. Li, Y. Chen, Some generalized neutrosophic number Hamacher aggregation operators and their application to group decision making, Int. J. Fuzzy Syst. 16 (2) (2014) 242-255.

[38] H. Ma, Z.G. Hu, User preferences-aware recommendation for trustworthy cloud services based on fuzzy clustering, J. Cent. South Univ. 22 (2015) 3495-3505.

[39] H. Ma, Z.G. Hu, Recommend trustworthy services using interval numbers of four parameters via cloud model for potential users, Front. Comput. Sci. 9 (6) (2015) 887-903.

[40] H. Ma, Z.G. Hu, L. Yang, T. Song, User feature-aware trustworthiness measurement of cloud services via evidence synthesis for potential users, J. Vis. Lang. Comput. 25 (6) (2014) 791-799.

[41] P. Majumdar, S.K. Samanta, On similarity and entropy of neutrosophic sets, J. Intell. Fuzzy Syst. 26 (3) (2014) 1245-1252.

[42] M. Mehdi, N. Bouguila, J. Bentahar, Probabilistic approach for QoS-aware recommender system for trustworthy web service selection, Appl. Intell. 41 (2) (2014) 503-524.

[43] M. Menzel, M. Schönherr, S. Tai, (MC2) 2: criteria, requirements and a software prototype for Cloud infrastructure decisions, Softw. Pract. Exp. 43 (11) (2013) 1283-1297.

[44] Y. Mo, J. Chen, X. Xie, C. Luo, L.T. Yang, Cloud-based mobile multimedia recommendation system with user behavior information, IEEE Syst. J. 8 (1) (2014) 184-193.

[45] H. Mouratidis, S. Islam, C. Kalloniatis, S. Gritzalis, A framework to support selection of cloud providers based on security and privacy requirements, J. Syst. Softw. 86 (9) (2013) 2276-2293.

[46] P.S. Pawar, M. Rajarajan, S.K. Nair, A. Zisman, Trust model for optimized cloud services, in: Trust Management VI, Springer, 2012, pp. 97-112.

[47] J.J. Peng, J.Q. Wang, J. Wang, L.J. Yang, X.H. Chen, An extension of ELECTRE to multi-criteria decision-making problems with multi-hesitant fuzzy sets, Inform. Sci. 307 (2015) 113-126.

[48] A.S. Prasad, S. Rao, A mechanism design approach to resource procurement in cloud computing, IEEE Trans. Comput. 63 (1) (2014) 17-30.

[49] G. Prestifilippo, D. Roy, J. Reipert, Real-time risk management through the use of intelligent cloud-computing solutions, Product. Manag. 19 (4)(2014)62-64.

[50] Princeton University, PlanetLab, February 2016. URL: http://www.planetlab.org.

[51] C. Qu, R. Buyya, A cloud trust evaluation system using hierarchical fuzzy inference system for service selection, in: 2014 IEEE 28th International Conference on Proc. of Advanced Information Networking and Applications, AINA, IEEE, 2014, pp. 850-857.

[52] A. Ramaswamy, A. Balasubramanian, P. Vijaykumar, P. Varalakshmi, A mobile agent based approach of ensuring trustworthiness in the cloud, in: Proc. of 2011 International Conference on Recent Trends in Information Technology, ICRTIT, IEEE, 2011, pp. 678-682.

[53] S. Ren, M. van der Schaar, Dynamic scheduling and pricing in wireless cloud computing, IEEE Trans. Mob. Comput. 13 (10) (2014) 2283-2292.

[54] D. Rosaci, G.M. Sarné, Recommending multimedia web services in a multidevice environment, Inf. Syst. 38 (2) (2013) 198-212.

[55] R. Şahin, M. Karabacak, A multi attribute decision making method based on inclusion measure for interval neutrosophic sets, Int. J. Eng. Appl. Sci. 2 (2) (2015) 13-15.

[56] P. Saripalli, B. Walters, Quirc: A quantitative impact and risk assessment framework for cloud security, in: Proc. of 2010 IEEE 3rd International Conference on Cloud Computing, CLOUD, IEEE, 2010, pp. 280-288.

[57] A. Sengupta, T.K. Pal, On comparing interval numbers, European J. Oper. Res. 127 (1) (2000) 28-43.

[58] A. Sengur, Y. Guo, Color texture image segmentation based on neutrosophic set and wavelet transformation, Comput. Vis. Image Underst. 115 (8) (2011) 1134-1144.

[59] H. Shen, G. Liu, An efficient and trustworthy resource sharing platform for collaborative cloud computing, IEEE Trans. Parallel Distrib. Syst. 25 (4) (2014) 862-875.

[60] T. Shinoj, S.J. John, Intuitionistic fuzzy multisets and its application in medical diagnosis, World Acad. Sci. Eng. Technol. 6 (1) (2012) 1418-1421.

[61] S. Silas, E.B. Rajsingh, K. Ezra, Efficient service selection middleware using ELECTRE methodology for cloud environments, Inf. Technol. J. 11 (7) (2012) $868-875$

[62] F. Smarandache, A unifying field in logics: neutrosophic logic, philosophy, (1999) 1-141.

[63] L. Sun, J. Ma, Y. Zhang, H. Dong, F.K. Hussain, Cloud-FuSeR: Fuzzy ontology and MCDM based cloud service selection, Future Gener. Comput. Syst. 57 (2016) 42-55. 
[64] V. Torra, Hesitant fuzzy sets, Int. J. Intell. Syst. 25 (6) (2010) 529-539.

[65] I.B. Turksen, Interval valued fuzzy sets based on normal forms, Fuzzy Sets and Systems 20 (2) (1986) 191-210.

[66] X. Wang, J. Cao, Y. Xiang, Dynamic cloud service selection using an adaptive learning mechanism in multi-cloud computing, J. Syst. Softw. 100 (2015) $195-210$

[67] H. Wang, F. Smarandache, R. Sunderraman, Y.-Q. Zhang, Interval Neutrosophic Sets and Logic: Theory and Applications in Computing: Theory and Applications in Computing, Hexis Phoenix Az, 2005.

[68] J.Q. Wang, J.T. Wu, J. Wang, H.Y. Zhang, X.H. Chen, Interval-valued hesitant fuzzy linguistic sets and their applications in multi-criteria decision-making problems, Inform. Sci. 288 (2014) 55-72.

[69] J.Q. Wang, J.T. Wu, J. Wang, H.Y. Zhang, X.H. Chen, Multi-criteria decisionmaking methods based on the Hausdorff distance of hesitant fuzzy linguistic numbers, Soft Comput. (2015) 1-13.

[70] G. Wang, C. Xu, D. Li, Generic normal cloud model, Inform. Sci. 280 (2014) 1-15

[71] Z.S. Xu, On method for uncertain multiple attribute decision making problems with uncertain multiplicative preference information on alternatives, Fuzzy Optim. Decis. Mak. 4 (2) (2005) 131-139.

[72] Z.S. Xu, Dependent uncertain ordered weighted aggregation operators, Inf. Fusion 9 (2) (2008) 310-316.

[73] C. Xu, G. Wang, Q. Zhang, A new multi-step backward cloud transformation algorithm based on normal cloud model, Fund. Inform. 133 (1) (2014) 55-85.

[74] J. Ye, Multicriteria decision-making method using the correlation coefficient under single-valued neutrosophic environment, Int. J. Gen. Syst 42 (4) (2013) 386-394.

[75] J. Ye, A multicriteria decision-making method using aggregation operators for simplified neutrosophic sets, J. Intell. Fuzzy Syst. 26 (5) (2014) 2459-2466.

[76] J. Ye, Similarity measures between interval neutrosophic sets and their applications in multicriteria decision-making, J. Intell. Fuzzy Syst. 26 (1)(2014) $165-172$.

[77] J. Yin, W. Lo, S. Deng, Y. Li, Z. Wu, N. Xiong, Colbar: A collaborative locationbased regularization framework for QoS prediction, Inform. Sci. 265 (2014) $68-84$

[78] C. Yu, L. Huang, Time-aware collaborative filtering for QoS-based service recommendation, in: Proc. of 2014 IEEE International Conference on Web Services, ICWS, IEEE, 2014, pp. 265-272.

[79] L. Yuchao, Z. Haisu, M. Yutao, L. Deyi, C. Guisheng, Collective intelligence and uncertain knowledge representation in cloud computing, China Commun. 8 (6) (2011) 58-66

[80] yunzhiliang.net, Quality evaluations of cloud, February 2016. URL: http://www.yunzhiliang.net/cloudtest/cloudtest.html.

[81] L. Zeng, B. Veeravalli, X. Li, SABA: A security-aware and budget-aware workflow scheduling strategy in clouds, J. Parallel Distrib. Comput. 75 (2015) $141-151$

[82] H.Y. Zhang, P. Ji, J.Q. Wang, X.H. Chen, An improved weighted correlation coefficient based on integrated weight for interval neutrosophic sets and its application in multi-criteria decision-making problems, Int. J. Comput. Intell. Syst. 8 (6) (2015) 1027-1043.

[83] L. Zhang, C. Qing, Hybrid-context-aware web service selection approach, J. Internet Technol. 14 (1) (2013) 57-69.

[84] H.Y. Zhang, J.Q. Wang, X. Chen, An outranking approach for multi-criteria decision-making problems with interval-valued neutrosophic sets, Neural Comput. Appl. (2015) http://dx.doi.org/10.1007/s00521-00015-01882-00523.

[85] M. Zhang, L. Zhang, H. Cheng, A neutrosophic approach to image segmentation based on watershed method, Signal Process. 90 (5) (2010) 1510-1517.

[86] Y.L. Zhang, Z.B. Zheng, M.R. Lyu, WSPred: A time-aware personalized OoS prediction framework for Web services, in: Proc. of 2011 IEEE 22nd International Symposium on Software Reliability Engineering, ISSRE, IEEE, 2011, pp. 210-219.

[87] Z.B. Zheng, H. Ma, M.R. Lyu, I. King, Collaborative web service QoS prediction via neighborhood integrated matrix factorization, IEEE Trans. Serv. Comput. 6 (3) (2013) 289-299.
[88] Z. Zheng, Y. Zhang, M.R. Lyu, Distributed OoS evaluation for real-world web services, in: Proc. of 2010 IEEE International Conference on Web Services, ICWS, IEEE, 2010, pp. 83-90.

[89] Y. Zhong, Y. Fan, K. Huang, W. Tan, J. Zhang, Time-aware service recommendation for mashup creation, IEEE Trans. Serv. Comput. 8 (3) (2015) 356-368.

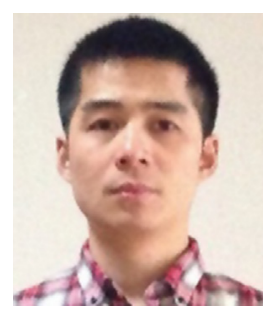

Hua Ma received his B.S. degree in computer science and technology and M.S. degree in computer application technology from Central South University, Changsha, China in 2003 and in 2006, respectively. His research interests focus on cloud computing, trusted computing and high performance computing. He is currently a Ph.D. candidate from Central South University, Changsha, China. He is a member of the IEEE.

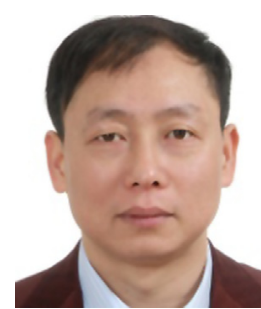

Zhigang Hu received his B.S. degree and M.S. degree from Central South University, Changsha, China in 1985 and in 1988, and received his Ph.D. degree from Central South University, Changsha, China in 2002. His research interests focus on the parallel computing, high performance computing and energy efficiency of cloud computing. He is currently a professor and Ph.D. supervisor of Central South University, Changsha, China.

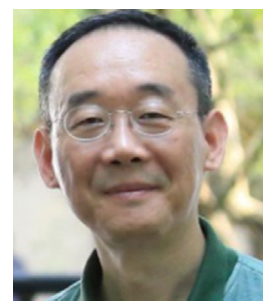

Keqin Li is a SUNY Distinguished Professor of computer science in the State University of New York. He is also a Distinguished Professor of Chinese National Recruitment Program of Global Experts ( 1000 Plan) at Hunan University and National Supercomputing Center in Changsha, China. He has published over 410 journal articles, book chapters, and refereed conference papers, and has received several best paper awards. He is currently or has served on the editorial boards of IEEE Transactions on Parallel and Distributed Systems, IEEE Transactions on Computers, IEEE Transactions on Cloud Computing, and Journal of Parallel and Distributed Computing. He is an IEEE Fellow.

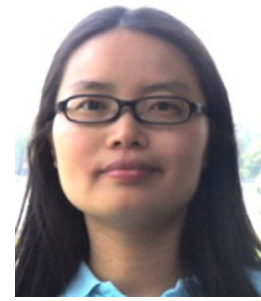

Hongyu Zhang received her M.S. degree in computer software and theory from the School of Information Science and Engineering, Central South University, Changsha, China, in 2005 and Ph.D. degree in management science and engineering from the Business School, Central South University, Changsha, China, in 2009. Her research interests include the multi-criteria decision-making and its applications. She is currently an associate professor with the Business School, Central South University, Changsha, China. 